\title{
Monitoring and analysis of ocean swell fields from space: New methods for routine observations
}

\author{
Fabrice Collard, ${ }^{1}$ Fabrice Ardhuin, ${ }^{2}$ and Bertrand Chapron $^{3}$ \\ Received 29 November 2008; revised 21 February 2009; accepted 21 April 2009; published 22 July 2009.
}

[1] Satellite synthetic aperture radar (SAR) observations can provide a global view of ocean swell fields when using a specific "wave mode" sampling. A methodology is presented to routinely derive integral properties of the longer-wavelength (swell) portion of the wave spectrum from SAR level 2 products and both monitor and predict their evolution across ocean basins. SAR-derived estimates of swell height and energy-weighted peak period and direction are validated against buoy observations, and the peak directions are used to project the peak periods in one dimension along the corresponding great circle route, both forward and back in time, using the peak period group velocity. The resulting real-time data set of great circle-projected peak periods produces two-dimensional maps that can be used to monitor and predict the spatial extent and temporal evolution of individual ocean swell fields as they propagate from their source region to distant coastlines. The result is found to be consistent with the dispersive arrival of peak swell periods at a midocean buoy. The simple great circle propagation method cannot project the swell heights in space like the peak periods, because energy evolution along a great circle is a function of the source storm characteristics and the unknown swell dissipation rate. A more general geometric optics model is thus proposed for the far field of the storms. This model is applied here to determine the attenuation over long distances. For one of the largest recorded storms, observations of $15 \mathrm{~s}$ period swells are consistent with a constant dissipation rate that corresponds to a $3300 \mathrm{~km}$ e-folding scale for the energy. In this case, swell dissipation is a significant term in the wave energy balance at global scales.

Citation: Collard, F., F. Ardhuin, and B. Chapron (2009), Monitoring and analysis of ocean swell fields from space: New methods for routine observations, J. Geophys. Res., 114, C07023, doi:10.1029/2008JC005215.

\section{Introduction}

[2] Storms over the ocean produce long surface gravity waves that propagate as swell out of their generation area. In deep water, the wave phase speed $C$ and period $T$ are proportional. As the phase speed of the dominant waves $C_{p}$ does not exceed 1.2 times the wind speed at $10 \mathrm{~m}$ height $U_{10}$ [Pierson and Moskowitz, 1964], the longest-period waves must be generated by very intense winds. For example, the generation of waves of period $T$ larger than $16 \mathrm{~s}$ requires winds with speeds over $18 \mathrm{~m} \mathrm{~s}^{-1}$ blowing over a distance of the order of $1000 \mathrm{~km}$, to produce a significant energy, or yet stronger winds over a shorter fetch [Munk et al., 1963]. Such a large region of high winds is generally associated with a smaller storm center from which the long swells radiate. Waves further evolve after their generation with an important transfer of energy toward both high and

\footnotetext{
${ }^{1}$ Division Radar, Collecte Localisation Satellites, Plouzané, France.

${ }^{2}$ Service Hydrographique et Océanographique de la Marine, Brest, France.

${ }^{3}$ Laboratoire d'Océanographie Spatiale, Centre de Brest, Ifremer, Plouzané, France.
}

Copyright 2009 by the American Geophysical Union. 0148-0227/09/2008JC005215\$09.00 low frequencies, because of nonlinear wave-wave interactions. Away from that core, nonlinear interactions become negligible [Hasselmann, 1963] and long-period swells have been observed to propagate over very large distances, up to halfway around the globe [Munk et al., 1963], radiating a large amount of momentum and energy across ocean basins. This measurable long-distance propagation is made possible by a limited loss of energy.

[3] The wave field at any time $t$, latitude $\phi$ and longitude $\lambda$, is described by its local two-dimensional spectral densities function $G(f, \theta)$ with the frequency $f$ and direction $\theta$. In the limit of geometrical optics, the spectral density is radiated at the group speed $C_{g}$ in the direction of wave propagation, and can be expressed as a function of $G$ at any previous time $t_{0}$. Allowing for a spatial decay at a rate $\mu$, the spectral energy balance is [e.g., Munk et al., 1963]

$$
G(t, \phi, \lambda, f, \theta)=G\left(t_{0}, \phi_{0}, \lambda_{0}, f, \theta_{0}\right) \exp \left(\int_{t_{0}}^{t}-\mu C_{g} \mathrm{~d} t\right) .
$$

In deep water without current, the initial position $\phi_{0}, \lambda_{0}$ and direction $\theta_{0}$ are given by following the great circle that goes through the point of coordinates $\phi, \lambda$ with a direction $\theta+\pi$ over a distance $X=\left(t-t_{0}\right) C_{g}=\left(t-t_{0}\right) g /(4 \pi f)$. This 
corresponds to a spherical distance $\alpha=X / R$ along the great circle, where $R$ is the Earth radius.

[4] Equation (1) can be used to invert $\mu$ from wave measurements. For swell periods shorter than $13 \mathrm{~s}$, Snodgrass et al. [1966] have measured an e-folding scale $L_{e}=1 / \mu=$ $5000 \mathrm{~km}$ (this number corresponds to a $0.1 \mathrm{~dB} \mathrm{deg}^{-1}$ attenuation in their analysis). For larger periods, Snodgrass et al. [1966] could only conclude that $L_{e}$ is larger, possibly infinite. In the past 40 years, little progress has been made on these conclusions [Waves in Shallow Water Environment (WISE) Group, 2007]. Yet this question if of high practical importance, either for wave forecasting [e.g., Rascle et al., 2008] or other geophysical investigations regarding air-sea fluxes or microseismic noise [e.g., Grachev and Fairall, 2001; Kedar et al., 2008].

[5] A theoretical upper bound for $L_{e}$ is given by the viscous theory (see also Appendix A for a simple derivation [Dore, 1978]). According to theory, the largest shears are found right above the water surface, and the air viscosity dominates the dissipation of swells, giving, in deep water,

$$
L_{e, \text { max }}=\frac{\rho_{w} g^{2}}{4 \rho_{a} \sigma^{3} \sqrt{2 \nu_{a} \sigma}},
$$

where $\nu_{a}$ is the air viscosity, $\sigma=2 \pi F=2 \pi / T$. For $T=13 \mathrm{~s}$ this gives $L_{e, \max }=45,000 \mathrm{~km}$, which means that over a realistic propagation distance of $10,000 \mathrm{~km}$ the energy of 13 s swells is only reduced by $25 \%$.

[6] Swells are thus expected to be very consistent over distances that are only limited by the size of ocean basins. The analysis of swells at this global scale should provide insights into their dynamics, including propagation and dissipation, but also into the structure of the generating areas, in a way similar to the use of the cosmic microwave background for the analysis of the early universe.

[7] The present paper provides two important intermediate steps toward that goal. First, we demonstrate in section 2 how sparse data from a single spaceborne synthetic aperture radar can be combined dynamically to provide a consistent picture of swell fields. This internal consistency reveals the quality of the synthetic aperture radar (SAR)-derived data set which we further verify quantitatively with buoy data. In section 3, we discuss and derive the asymptotic far-field swell energy evolution. Numerical investigations are performed to check the validity of the asymptotic solutions. This result provides a tool to interpret measured swell heights in terms of propagation and dissipation. This method is illustrated with one example that corresponds to a strong swell dissipation. Conclusions follow in section 4.

\section{Space-Time Consistency of Spaceborne Swell Observations}

[8] Investigations by Holt et al. [1998] and Heimbach and Hasselmann [2000] have shown that spaceborne synthetic aperture radar (SAR) data can be used to image the same swell field over 3 to 10 days as it propagates along the ocean surface. These preliminary studies have shown that the combination of SAR data at different places and times yields a position of the generating storm, and predictions for the arrival time of swells with different periods and directions. Heimbach and Hasselmann [2000] have further pointed to shortcomings in the wind provided by an atmospheric circulation model for a given Southern Ocean storm, on the basis of systematic biases in wind-forced wave model results compared to SAR observations. Unfortunately, the systematic analysis of such data has been very limited, and generally confined to data assimilation in wave forecasting models [e.g., Hasselmann et al., 1997; Aouf et al., 2006]. This narrow use of SAR data is due to three essential difficulties.

[9] First, a SAR image is not a picture of the ocean surface and the relationship between the spectrum of the SAR image and that of the ocean surface elevation is nonlinear and fairly complex [e.g., Krogstad, 1992]. Sophisticated methods have been developed in order to estimate the surface elevation spectrum [e.g., Hasselmann et al., 1996; Schulz-Stellenfleth et al., 2005]. These methods had to be implemented by the user of the data, and generally required some a priori first guess of the wave field provided by a numerical model. For longer wave systems, the imaging mechanisms are essentially quasi-linear, making possible a simpler methodology used by the European Space Agency (ESA) to generate a level 2 (L2) product. The method is fully described by Chapron et al. [2001]. It uses no outside wave information, and builds on the use of complex SAR data developed by Engen and Johnsen [1995] to remove the $180^{\circ}$ directional ambiguity in wave propagation direction. The quality of the $\mathrm{L} 2$ data has been repeatedly analyzed [e.g., Johnsen et al., 2006; Collard et al., 2005]. Because long ocean swells have large wavelengths and smaller steepnesses, the L2 products corresponding to this spectral range have higher relative quality, confirming that the imaging mechanism is well described under the quasi-linear assumption.

[10] All SAR data used here are such L2 products, provided by ESA and obtained with the L2 processor version operational since November 2007, and described by Johnsen and Collard [2004]. The data for times before that date were reprocessed with this same processor. In previous real-time data, frequent low-wave number artifacts were caused by insufficient filtering of nonwave signatures in the radar images. This filtering is necessary to remove the contributions of atmospheric patterns or other surface phenomena like ships, slicks, sea ice, or islands, with spectral signatures that can overlap the swell spectra. The L2 product contains directional wave spectra with a resolution of $10^{\circ}$ in directions and an exponential discretization in wave numbers spanning wavelengths of 30 to $800 \mathrm{~m}$ with 24 exponentially spaced wave numbers, corresponding to wave periods with a $7 \%$ increment from one to the next.

[11] The second practical problem is that the data obtained from an orbiting platform are sparse and with a sampling that makes a direct analysis difficult. Hereafter, we show that the space-time consistency of the swell field can be used to fill in the gaps in the observations and produce continuous observations of swell periods and directions in space and time.

[12] Third, and last, for a simple use of SAR data, parameters that are not affected by the variable resolution in SAR scenes [e.g., Kerbaol et al., 1998] are desirable. Schulz-Stellenfleth et al. [2007] have proposed to produce parameters representing the entire sea state by extending the 


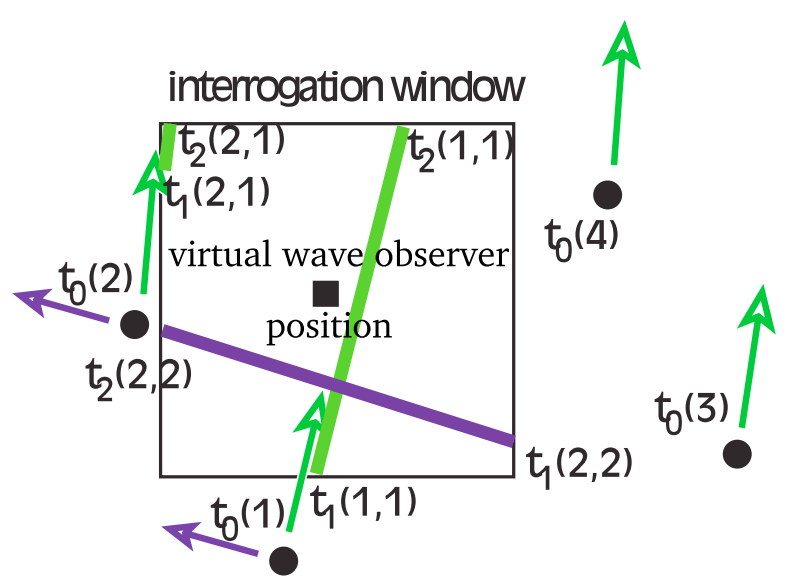

Figure 1. Schematic definition of a virtual wave observer. Any SAR observation $i$ is available at a time $t_{0}(i)$ on the black dots. All swell partitions $(i, j)$ (here indicated by the arrows) are propagated and may cross the interrogation window from time $t_{1}(i, j)$ to $t_{2}(i, j)$.

resolved spectrum with an empirical windsea contribution. Here we take the opposite approach and restrict the resolved part of the spectrum by using a spectral partitioning (see Appendix B for details) to retrieve the swell significant wave height $H_{s s}$, defined as four times the square root of the energy of one swell system, and the peak period $T_{p}$ and peak directions $\theta_{p}$. Thus one SAR typically produced one or two swell parameters for distinct swell systems.

[13] For $H_{s S}$, only very limited validations have been performed [Collard et al., 2006]. We thus perform a thorough analysis of SAR-derived $H_{S S}$ comparing to colocated buoy data (see Appendix B for details).

[14] The bias on $H_{s S}$ derived from ESA level 2 products is found to be primarily a function of the swell height and wind speed, increasing with height and decreasing with wind speed. Variations in standard deviation are dominated by the swell height and peak period, with the most accurate estimations for intermediate periods of 14 to $17 \mathrm{~s}$. For wind speeds in the range 3 to $8 \mathrm{~m} \mathrm{~s}^{-1}, H_{s s}$ has a bias of $0.24 \mathrm{~m}$ and the standard deviation of the errors is $0.29 \mathrm{~m}$.

[15] We thus corrected the values of $H_{s s}$ by subtracting a bias model given by

$$
b_{h}=0.11+0.1 H_{S S}-0.1 \max \left\{0, U_{10 S A R}-7\right\}
$$

where $H_{S S}$ is in meters and the wind speed $U_{10 S A R}$ is in $\mathrm{m}$ $\mathrm{s}^{-1}$. From now on, all the reported values of $H_{S S}$ will be corrected using this expression. After correction, the standard deviation of $H_{s s}$ estimates is reduced to less than

$$
\sigma_{h}=0.10+\min \left\{0.25 H_{s s}, 0.8\right\}
$$

where $\sigma_{h}$ and $H_{s s}$ are in meters.

[16] The quality of $T_{p}$ and $\theta_{p}$ has already been carefully studied and are routinely monitored. The root mean square (r.m.s.) error on $T_{p}$ is less than $10 \%$ of the measured value for $T_{p}>12 \mathrm{~s}$, and the r.m.s. error on $\theta_{p}$ is $22^{\circ}$ for these same swells [Johnsen and Collard, 2004], with little bias. Because few directional in situ measurements are available, we demonstrate here an original semiquantitative dynamical validation of these two parameters.

\subsection{Virtual Wave Observers}

[17] Given these SAR-derived estimates of $\theta_{p}$ and $T_{p}$, linear dispersion relationship and the principle of geometrical optics can then be exploited to predict arrival times and locations of the swell.

[18] In order to obtain swell conditions at the location of a "virtual wave observer," we define an interrogation window covering 2 by 2 degrees in latitude and longitude. According to the SAR-derived peak parameters, swell partitions from the entire ocean basin are propagated, both forward and backward, along great circles in space and time. These theoretical trajectories are followed with a constant group speed $g T_{p} /(4 \pi)$, starting off in direction $\theta_{p}$ from the observation point. From any observation time $t_{0}$, these great circles may cut through the interrogation window from times $t_{1}$ to $t_{2}$ (thick solid lines in Figure 1, with different colors for different partitions). As the maximum value of $\left|t_{2}-t_{0}\right|$ and $\left|t_{1}-t_{0}\right|$ is increased from $6 \mathrm{~h}$ to 6 days, the time evolution of the peak frequencies and peak directions at the virtual observer gradually reveals similar ridges to the one observed in real buoy measurements (Figure 2).

[19] In Figures $2 b-2 d$, each horizontal colored segment corresponds to one swell partition that crosses the spatial window between times $t_{1}$ and $t_{2}$. Some segments are very short, corresponding to trajectories that barely cut one corner of the window.

[20] Clearly the SAR detects the direction of the most energetic part of the wave spectrum measured by the buoys (Figure 2a). At frequencies above $0.1 \mathrm{~Hz}$, the virtual observer patterns appear rather noisy. Shorter scales are not so correlated. These shorter components are often observed as part of the wind sea for which the propagation with a single group speed and direction is not a good approximation. Also, propagated high-frequency swells, such as the $0.12 \mathrm{~Hz}$ waves coming from direction 200 on 10 July, do not show up in the real buoy record. This is possibly the result of a relatively high dissipation rate for these swells.

[21] For frequencies below $0.08 \mathrm{~Hz}$, the virtual observer shows ridge-like structures similar to those observed in situ because of the dispersive arrivals of swells from remote storms [e.g., Munk et al., 1963; Gjevik et al., 1988]. Even the faintest events are well detected, such as the $0.06 \mathrm{~Hz}$ arrival on 23 July, even though that $15 \mathrm{~s}$ swell of $0.5 \mathrm{~m}$ is dwarfed by a another $0.8 \mathrm{~m}$ and $12 \mathrm{~s}$ swell, and a $2 \mathrm{~m}$ and $8 \mathrm{~s}$ wind sea. Swell detection with the virtual observer reaches its limits when the swell height is very low, such as on 3 July with a $20 \mathrm{~s} 0.3 \mathrm{~m}$ swell well detected by the buoy (the green-orange ridge at $0.05 \mathrm{~Hz}$ ). Because we use a single trajectory emanating from one observed swell partition the relatively small interrogation window can easily be missed after $10,000 \mathrm{~km}$ of propagation.

[22] Our discrete propagation technique suffers from a randomized version of the "garden sprinkler effect" that, if not corrected for, can create unrealistic flower-like patterns 


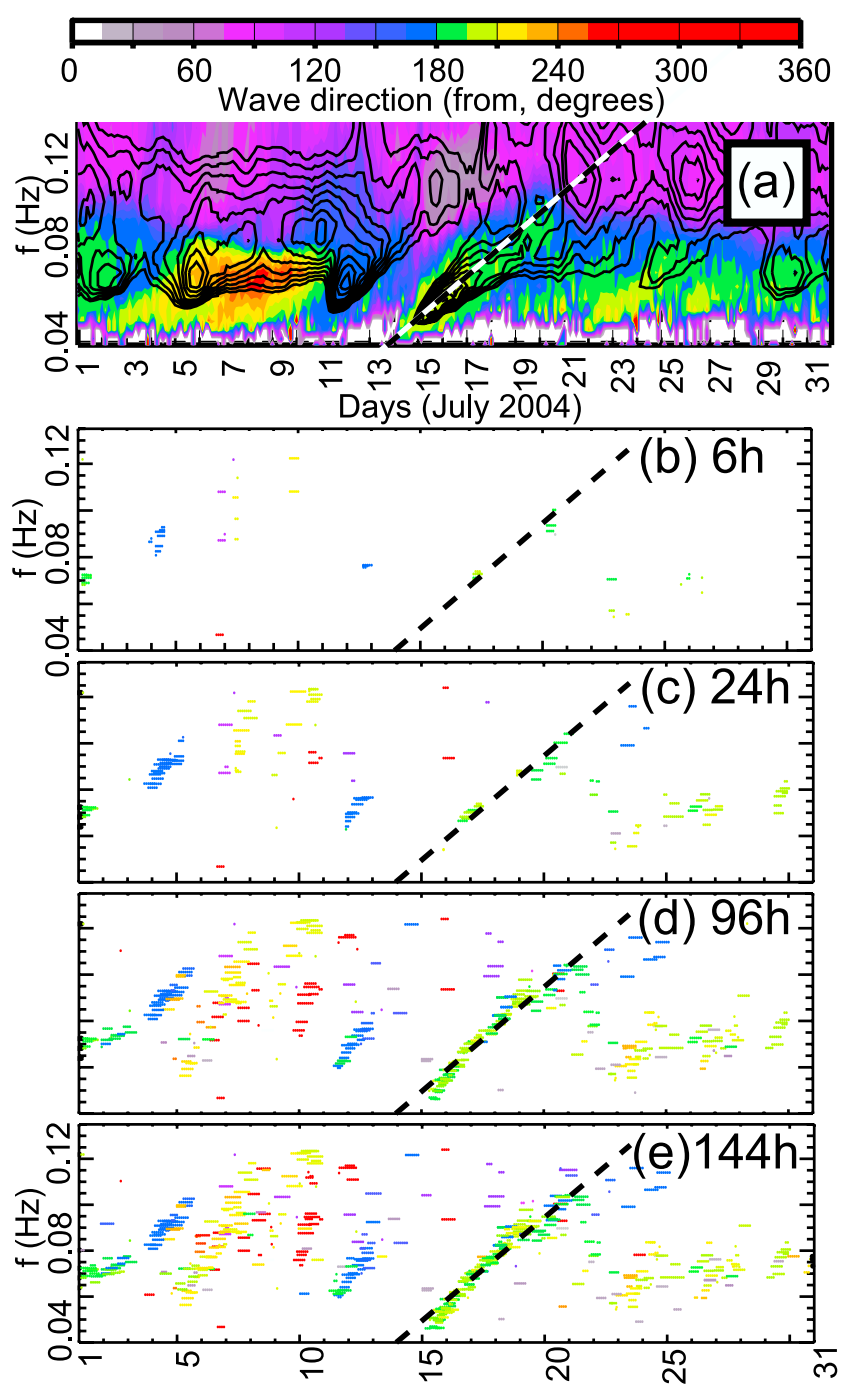

Figure 2. (a) Energy and mean direction spectrum measured in situ by the Christmas Island buoy (World Meteorological Organization (WMO) number 51028). Contours, equally spaced from 0.1 to 1.4 , indicate the natural logarithm of the spectral energy density $F(f)$. Colors indicate the mean arrival direction at each frequency. $(b-e)$ Peak direction (colors) as a function of time and peak frequency for swell partitions at a SAR virtual buoy located around the Christmas Island buoy (WMO number 51028). The maximum propagation time to produce the virtual buoy data is increased from $6 \mathrm{~h}$ (Figure 2b) to 6 days (Figure 2e). The sloping straight line fitted to the observed SAR ridge from 16 July at $0.05 \mathrm{~Hz}$ to 21 July at $0.105 \mathrm{~Hz}$ is the same as line in Figure $2 \mathrm{a}$ that corresponds to the buoy observation. This delayed arrival would correspond to a point source at $6100 \mathrm{~km}$ from Christmas Island.

in the far field of storms in numerical wave models that use a discretized spectrum [e.g., Tolman, 2002]. Our choice of a single group speed and direction, because a narrow swell spectrum is not resolved by the SAR, produces a discrete wave field (the dots in Figure 3). With the present processing this is smoothed by the finite size of our interrogation window (Figure 1). An extension of the present technique could use neighboring group speeds and directions to take into account the frequency and directional spread of the sea state, which would allow the use of a smaller window. Just like the estimation of propagated wave heights, discussed below, the estimation of a spectra width that cannot be resolved by the SAR may use some further information on the structure of the generating storm.

[23] Other errors in the present technique can also be attributed to the SAR processing. In particular, a maximum value is defined for the transfer function used to obtain the wave spectrum from the SAR image [Johnsen and Collard, 2004]. Although this is designed to prevent the amplification of measurement noise, long swells such as this $20 \mathrm{~s}$ event have very small slopes, and it is likely that they are underestimated in the wave spectrum because of this threshold in the processing.

[24] When propagated for 6 days, without any other information than the peak frequency and direction at the time of observations, the waves are remarkably consistent with the latest local observations. For the southern swells arriving at Christmas Island (Kiritimati, Kiribati) between 16 and 21 July (Figure 2), the difference in arrival times given by the virtual observer and real buoy is typically less than $12 \mathrm{~h}$. This is less than $10 \%$ of the maximum time between the SAR observation and the virtual observer record. This implies that the accuracy of the peak period estimate for each SAR partition must also be less than $10 \%$, consistent with previous validation studies [Johnsen and Collard, 2004; Johnsen et al., 2006]. The consistency of the arrival directions along the ridges also suggests that the root mean square (RMS) error in peak direction estimates must be close to $20^{\circ}$, comparable to the $22^{\circ}$ RMS difference between mean wave directions obtained from SAR wave mode and a numerical wave model for waves with periods longer than $12 \mathrm{~s}$ [Johnsen and Collard, 2004].

[25] Although it cannot replace the spectral resolving power of a buoy, the performance of the virtual observer is therefore comparable or better to that of human observers in terms of peak period and direction [Munk and Traylor, 1947]. The really missing bit is a wave height estimate along the swell propagation path. We will show that this may be obtained by estimating the source storm characteristics and the dissipation rate of swell energy.

\subsection{Storm Source Identification and "Fireworks"}

[26] Along the estimated trajectories, virtual observations can further be produced in a similar fashion. The animation of these propagated swells confirms the very well organized nature of storm swells crossing large ocean basins.

[27] From the relatively sparse and track-based initial satellite observation sampling, the swell persistency can then be used to capture fireworks patterns exploding from the few intense storms that occur over a period of several days (see Figure 3 and auxiliary material). ${ }^{1}$ In large ocean basins where swells are likely to be imaged several times by the same satellite, these fireworks can be used to estimate the time of arrival of swells from any given storm [e.g., Holt et al., 1998]. For this reason, these animations have been produced routinely every day since August 2007, for the

${ }^{1}$ Auxiliary materials are available in the HTML. doi:10.1029/ 2008JC005215 


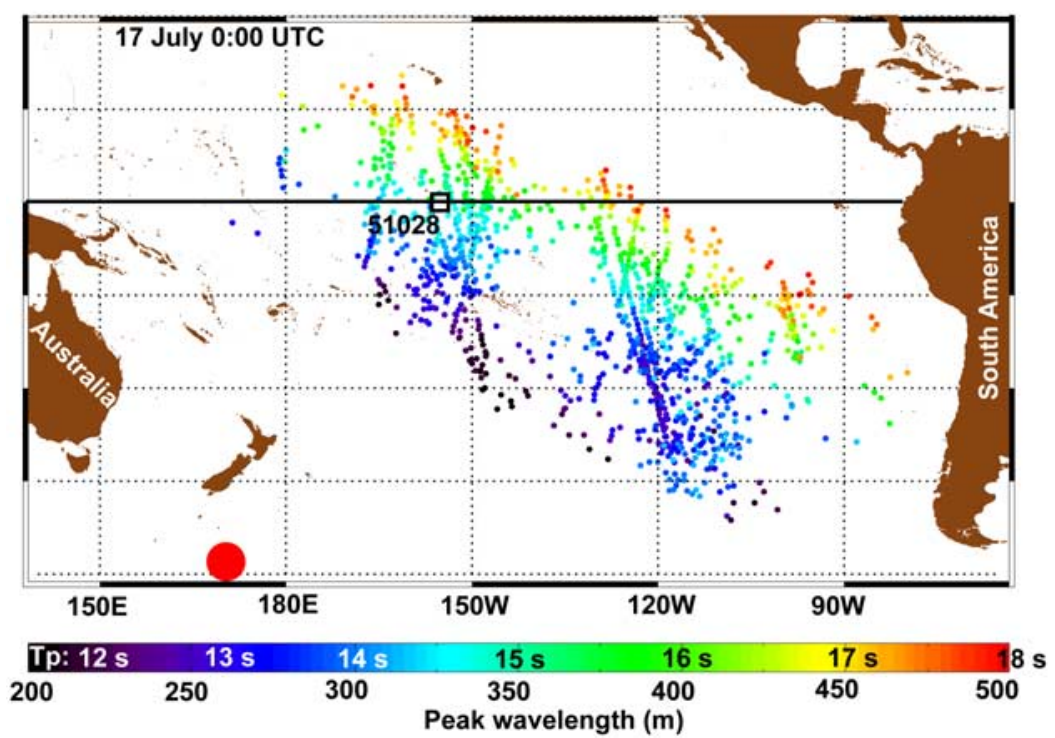

Figure 3. Snapshot of Animation S1 for 17 July 2004 at 0000 UTC. Each of the 1071 colored dots represents one observed swell partition, within 6 days of its observation, displaced along a great circle with the group speed corresponding to the detected peak period in the direction of the detected peak direction. Only swells with tracks that pass within $1000 \mathrm{~km}$ of the storm center (red disk) have been retained.

Pacific, Indian, and Atlantic oceans (see http://www.boosttechnologies.com/esa/images/, e.g., "nrt_pac.gif" for the Pacific Ocean).

[28] Using backward trajectories, the location and date of swell sources can further be defined as the spatial and temporal center of the convergence area and time of the trajectories. These positions have been verified to correspond to high wind conditions observed by scatterometers and reproduced by ECMWF wind analyses. We consider these storms to be the source of all the swell partitions that produce trajectories that pass within $12 \mathrm{~h}$ and $2000 \mathrm{~km}$ of their center. This processing, similar to the one performed by Heimbach and Hasselmann [2000], provides a global view of swell fields in both space and time, extending the coverage of similar techniques based on buoy data [Hanson and Phillips, 2001]. In Figure 4, a swell covers one Earth quadrant away from the storm, with a large detection gap that extends from the southern Pacific to California. This blank area is the long shadow cast by French Polynesia where wave energy is dissipated in the surf [e.g., Munk et al., 1963]. Observations were restricted to swell partitions with periods close to $17 \mathrm{~s}$, but the full data set typically covers swells with periods of 12 to $18 \mathrm{~s}$, as shown in Figure 3.

[29] The apparent self-consistency of both the virtual buoy plot (Figure 2d and Animation S1) are the result of the large autocorrelation length of the swell fields, which was expected from the in situ measurements of Darbyshire [1958], Munk et al. [1963], and Snodgrass et al. [1966]. Yet, these plots could not exhibit such patterns without a good accuracy of the SAR-derived peak periods and directions, used in the propagation methodology.

\section{Far-Field Swell Energy}

[30] All these illustrations of forward-backward ray tracing indicate the potential to use a simple Geometrical Optics
(GO) strategy. The next goal is then to determine the strength of the far-field radiated swell energy. This requires the definition of a swell source, and an estimation of the swell energy dissipation scale. For this we define the time $t_{0}$ as an initial condition after which there is no significant wave generation, or nonlinear evolution, for frequencies

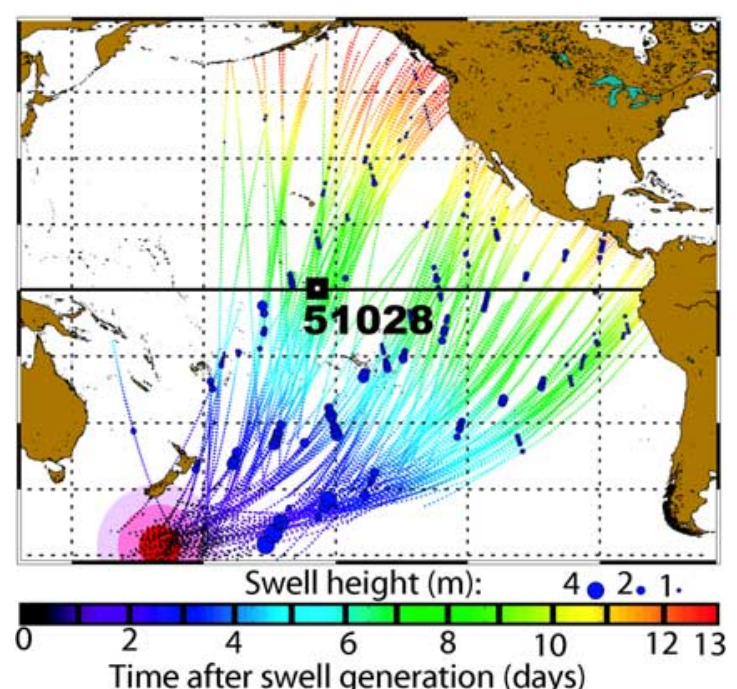

Figure 4. Finding the source storm. All swells with a $17 \pm$ $0.5 \mathrm{~s}$ period that were identified in 13 days of Envisat synthetic aperture radar data over the Pacific are refocussed from their location of observation (dots) following their direction of arrival at the theoretical group speed for $17 \mathrm{~s}$ waves. This focussing reveals a single swell generation event, well defined in space and time (pink to red disks). The back-tracking trajectories are color dated from black (9 July 20041800 UTC) to red (22 July 20041800 UTC). 

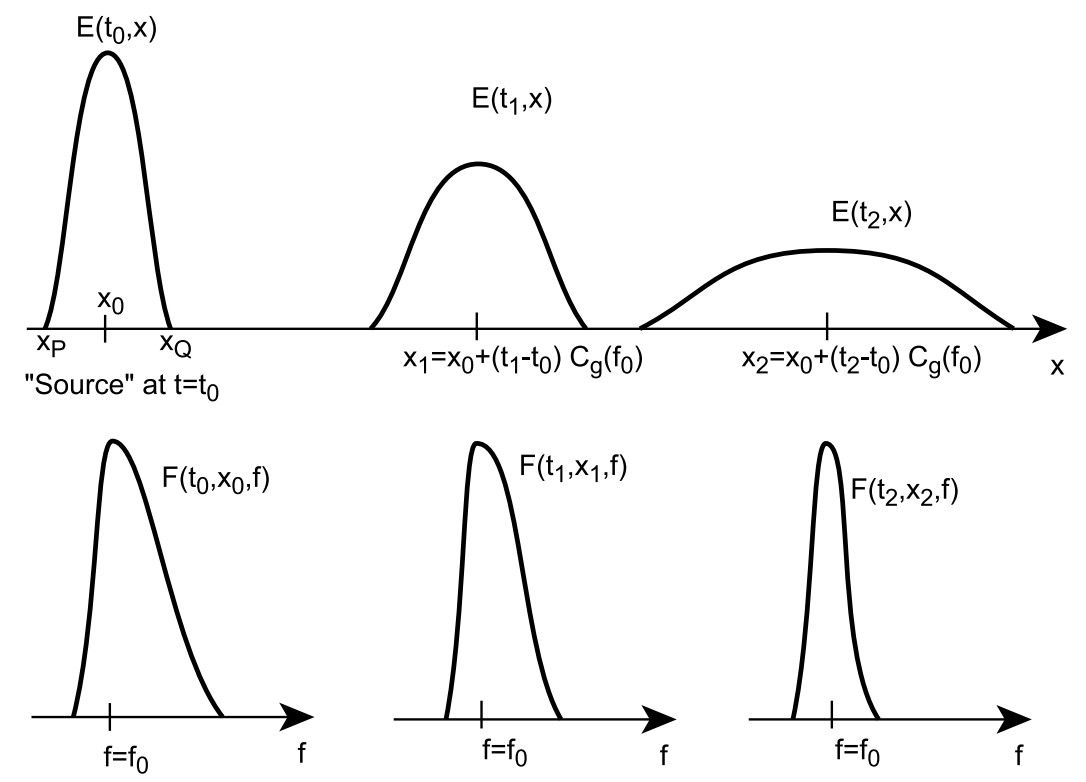

Figure 5. Dispersion of linear waves in one dimension. At any given time the spectrum is given by a propagation of the spectra at $t=t_{0}$. Taking $x_{1}=x_{0}+\left(t_{1}-t_{0}\right) C_{g}\left(f_{p}\right)$, the spectral density at $f_{p}$ is the same as for time $t_{0}$, but the spectrum is narrower, which gives a smaller elevation variance, $E\left(t_{1}, x_{1}\right)<E\left(t_{0}, x_{0}\right)$.

less than $f_{\max }$. Namely, at $t_{0}$ all the wave components with smaller frequencies have already been generated, so that the radiation of these waves is essentially fossil and fully governed by geometrical optics. The possible effects of diffraction and scattering are discussed by Munk et al. [1963], and, together with dissipation, will cause deviations from the G.O. model outlined below. We therefore make no restrictive hypothesis on what happens before $t_{0}$, and thus the motion of the generating storm has no direct effect on our results, but it obviously modifies the spatial distribution of the energy at $t_{0}$, which will be relevant.

[31] In reality, swells evolve over the course of their propagation as the result of their interactions with the local winds, mutual wave-wave interactions, interactions with other wave systems, including the local wind sea. Swells are also expected to evolve according to interactions with other oceanic motions that affect the upper ocean, namely surface currents, internal waves [e.g., Kudryavtsev, 1994] and turbulence. Depth and island scattering effects must also carefully be taken into account [Snodgrass et al., 1966; WISE Group, 2007]. Compared to these different mechanisms, frequency dispersion and angular spreading effects are certainly the first leading order phenomena to take into account for the major part of the decrease in the height of the swell systems. Indeed, as the long swell systems will be characterized by relatively small steepness parameters, nonlinear mutual wave-wave interactions do not appear to be important in scattering surface wave energy for distances more than a few storm radii outside the active generating area [Hasselmann, 1963]. Furthermore, the level of turbulence in the ocean does not appear to significantly affect the waves [Fabrikant and Raevsky, 1994; Ardhuin and Jenkins, 2006], and the conversion of surface wave energy into internal gravity wave energy by wave-wave interactions does not seem to be a leading order sink term for the energy balance of surface gravity waves. Finally, for the very long swell components, surface current refraction effects, proportional to the ratio between vertical current vorticity and the group velocity, may also be considered marginal.

[32] Away from island obstructions, the ratio between the angular width of swell arrivals and the mean spread in the generating area is approximately proportional to $1 / \sin \alpha$ with $\alpha$ the spherical distance from the storm. This approximation applies to large distances, and relatively small source regions. Closer to the source, the approximation does not hold. Swell amplitudes radiating from large extended sources will decrease more slowly than swell amplitudes emanating from compact sources. This transversal dispersion is associated to a narrowing of the directional spectrum $G$, for $\alpha<\pi / 2$, and a broadening for larger distances. The change in spectral density $F$ defined by

$$
F(t, \phi, \lambda, f)=\int_{0}^{2 \pi} G(t, \phi, \lambda, f, \theta) d \theta
$$

follows the spatial expansion (close to the source) and contraction (as waves approach the antipodes for $\alpha>\pi / 2$ ) of the energy front.

[33] Moreover, we can represent swell waves as a linear superposition of harmonic waves in narrow spectral band. Quite naturally, through the method of stationary phase, the group velocity is defined and the slowly varying wave envelope is found to decay. This decay is inversely proportional to the square root of the distance (Figure 5). Accordingly, far away from the generating sources, and in the absence of dissipation, the swell energy

$$
E_{s}(t, \phi, \lambda)=\int_{0}^{\infty} F(t, \phi, \lambda, f) \mathrm{d} f .
$$



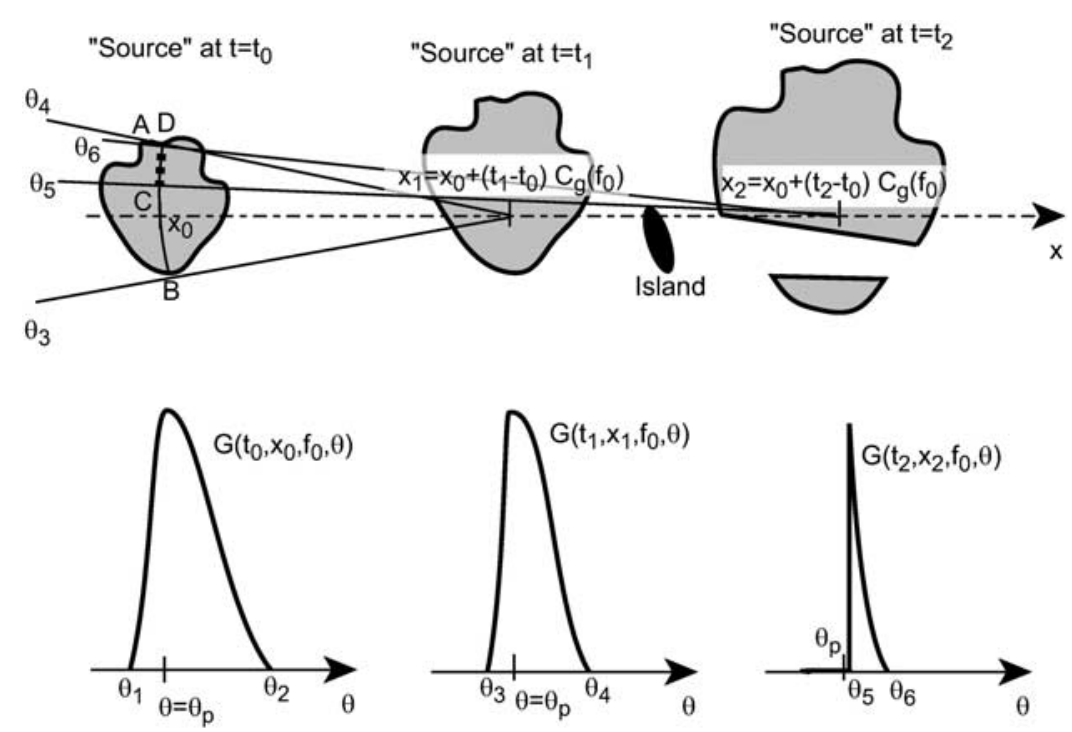

Figure 6. (top) Dispersion of linear waves in two dimensions, represented here on a flat surface for simplicity. The variable $y$ has been dropped as the spectra shown here are all at $y=0$. We call "source" the region of the ocean where waves with frequencies smaller than $f_{\min }$ can be found. As time goes by, the source expands in space because of both frequency dispersion (like in Figure 5) and geometrical dispersion. The wave energy with frequency $f_{0}$ that will be observed at points $x_{1}$ (at time $t_{1}$ ) and $x_{2}$ (at time $t_{2}$ ) is, at time $t_{0}$, along the thin arc circle $\mathrm{AB}$ and the thick dotted arc circle $\mathrm{CD}$, respectively. Because of the small island between $x_{1}$ and $x_{2}$, the energy that would have been recorded at $x_{2}$, if the island were not present, is actually dissipated on the shore of the island. As a result the local energy density $E\left(x_{2}\right)$ is reduced. At frequency $f_{0}$, contributions to $E\left(x_{2}\right)$ only come from angles $\theta_{5}$ to $\theta_{6}$. (bottom) The directional spectra are thus affected by the blocking effect of islands and the directional narrowing as one goes farther from $x_{0}$ (on the Earth this narrowing reverses after 10,000 km of propagation, because of the sphericity).

should decrease asymptotically like $1 /(\alpha \sin \alpha)$ when following a wave group (see Appendix $\mathrm{C}$ for a detailed proof).

\subsection{Snodgrass et al.'s [1966] Method}

[34] Using measurements with a limited or no directional resolution, Snodgrass et al. [1966] assumed that wave propagation was completely blocked by waters shallower than 60 fathoms (approximately $110 \mathrm{~m}$ ), and that diffraction could be neglected. For example, in Figure 6, the island would be represented by the 60 fathom depth contour. These authors then estimated a loss of swell energy from the deviation of the ratio of directionally integrated spectra, e.g., $F\left(x_{2}, f_{0}\right) / F\left(x_{1}, f_{0}\right)$, compared to what is expected from the lateral $1 / \sin \alpha$ dispersion effect, taking into account islands. Currents, shallow water areas and diffraction effects around islands are neglected here. These effects are discussed by Snodgrass et al. [1966].

[35] Rigorously, their method is inexact because the recording stations $x_{1}$ and $x_{2}$ measure wave groups that had neither exactly same propagation directions nor the same position when they were near $x_{0}$. Yet, because the wave field in the neighborhood of $x_{0}$ is the superposition of many independent wave trains, one can assume that the spectral density $G$ is a smooth function of the direction. Then we may say that over the intervals $\theta_{3}$ to $\theta_{4}$ or $\theta_{5}$ to $\theta_{6}, G$ does not change so much, i.e., in Figure $6, G\left(t_{0}, \phi_{D}, \lambda_{D}, f, \theta_{6}\right) \simeq$
$G\left(t_{0}, \phi_{D}, \lambda_{D}, f, \theta_{5}\right)$. On the sphere the application of equations (1) and (5) yields

$$
F\left(t, \phi, \lambda, f_{0}\right)=\frac{1}{\sin \alpha} \int_{C}^{D} G\left(t_{0}, \phi, \lambda, f_{0}, \theta_{0}\right) \frac{\mathrm{d} s}{R}\left[1+O\left(\frac{1}{\sin \alpha}\right)\right]
$$

where the integral is performed over the line segment joining $C$ to $D$. The error relative to the asymptote is the sum of two terms. One is proportional to the spatial gradient of $F$, because of the change from the arc circle to the segment, and the other corresponds to the relative variation of $G$ with $\theta$ over the range $\theta_{5}$ to $\theta_{6}$, which is small in the far field, provided that the directional spectrum is smooth enough.

[36] Under these two smoothness assumptions, and for large $\sin (\alpha)$, the ratios of spectral densities $F$ at $x_{1}$ and $x_{2}$, as used by Snodgrass et al. [1966], can be compared to the conservative equation (7) and used to diagnose the dissipation of swell energy. This does not apply if $x_{1}$ is in the vicinity of the storm or its antipode, where the observed arrival direction span a large range.

[37] In practice, this method can be very sensitive to the correct estimation of the island shadowing. For that reason, the measurement route chosen by Snodgrass et al. [1966] was far from ideal. Because they needed land to install most recording stations for the wave measurements, they used an almost north-south great circle that extends from the south 


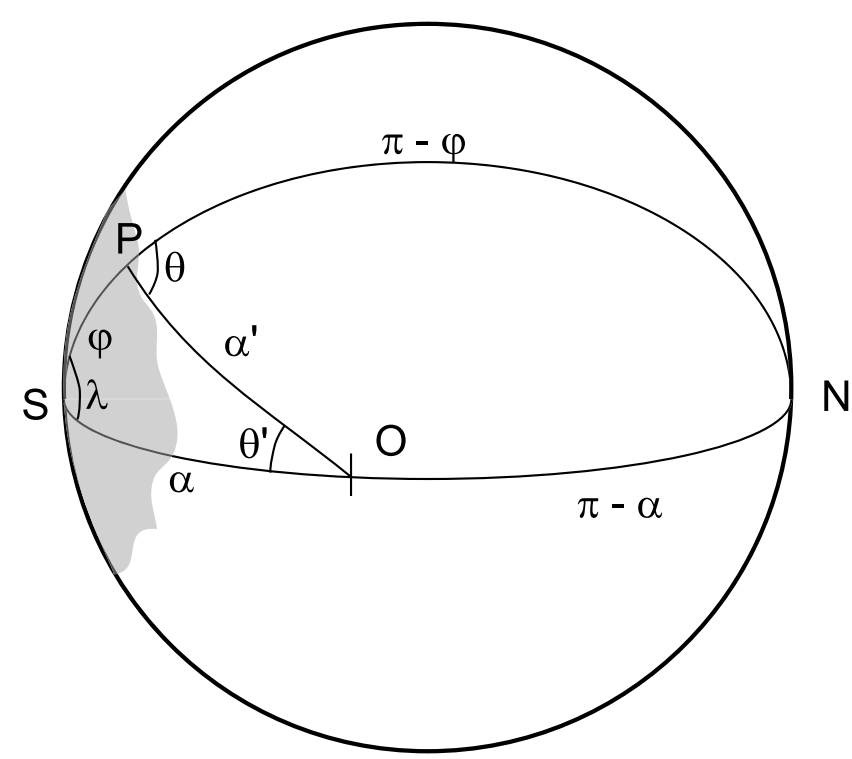

Figure 7. Geometry of the "fossil" swell field distribution at time $t_{0}=0$ (shaded area) and observation conditions. Any point $P$ of colatitude $\varphi$ and longitude $\lambda$ inside of the storm generates waves that are observed at point $O$. At time $t$ the observed waves that come from $P$ have a well-defined frequency given by equation $(\mathrm{C} 2)$, the function of the spherical distance $\alpha^{\prime}$ between $P$ and $O$, and a well-defined direction $\theta$ at $P$ relative to the north, which gives a direction $\pi-\theta^{\prime}$ at $O$. In the triangle $O P S$ the angles $\lambda, \theta^{\prime}$, and $\pi-\theta$ are related to the distances $\alpha^{\prime}, \varphi$, and $\alpha$ by the usual spherical trigonometry relationships, e.g., equation (C4).

of New Zealand (Cape Palliser) to Alaska (Yakutat), a route peppered with islands in its southern part, and partially blocked by the Hawaiian chain in its northern part. Also, storms typically refuse to line up with any measurement array. Their predefined great circle, although designed to follow a typical southern winter swell propagation path, always deviated by some extent form the actual track followed by the most energetic swells they recorded. For the Indian ocean storms, this difficulty was reduced by the relatively narrow range of angles that allows propagation from the Indian to the Pacific ocean.

\subsection{A Method Using Global Swell Heights}

[38] Now using an instrument with a global coverage, we can carefully avoid both problems by choosing propagation paths far away from the smallest island, and by exploiting only observations well aligned with the storms. However, because of the limited spectral resolution inherent to the SAR wave mode image size and processing, we cannot use the spectral distribution $G$ or $F$ of the energy, and can only use the energy $E_{s}$ integrated over a swell partition. We thus need for $E_{S}$ the equivalent of equation (7) for $F$.

[39] For simplicity, and without loss of generality, we take the source storm centered at time $t_{0}=0$ on the pole $S$ defined by a colatitude $\varphi=\phi-\pi / 2=0$, so that the distance from the storm center is $r=R \varphi$. We consider the swell energy $E_{S}$ observed at a position defined by the spherical distance $\alpha$ and we take the reference meridian to be in the direction of the observation point (Figure 7).
[40] We will later assume that the source area is relatively small with a size $R \Delta_{\alpha}$, where $\Delta_{\alpha}$ is the maximum value taken by $\varphi$ (Figure 7 ). In all the following derivations, we have chosen a fixed frequency $f_{0}$ and we follow a wave group of that frequency. The time of observation $t$ is thus related to $\alpha$ by $t=R \alpha / C g\left(f_{0}\right)$, so that the variable $t$ will be omitted.

[41] In Appendix C, we prove and verify that, in the absence of dissipation the swell energy $E_{s}$ decreases like $1 /(\alpha \sin \alpha)$ for large values of $\alpha$, and that typical storms should produce swells within $20 \%$ of this asymptote at distances larger than $4000 \mathrm{~km}$ from the storm center. A much larger observed deviation should thus reflect a gain or loss of energy by the propagating swells. The expected departures from the asymptotic evolution should be compared to those due to swell dissipation or generation. Even with perfect SAR observations, this is the intrinsic limit of the present method. A $20 \%$ error in energy conserving conditions may be misinterpreted as a dissipation or generation with an e-folding scale of the order of $20,000 \mathrm{~km}$, which gives a $20 \%$ energy change as waves propagate from 4000 to $8000 \mathrm{~km}$ away from the storm source.

\subsection{Illustration}

[42] To illustrate the method described above, we analyze of one of the most powerful swell field recorded over the past 4 years by Envisat's advanced synthetic aperture radar. The swell case illustrated in Figure 4 is not well suited because of the islands in the south-north swell tracks and the poor sampling of Envisat for the tracks going northeast from the storm, we have thus chosen another source, found on 12 February 2007 at 1800 UTC, and located at $168 \mathrm{E}$ and $38 \mathrm{~N}$. This swell was generated by a storm moving eastward with maximum westerly winds of $26 \mathrm{~m} \mathrm{~s}^{-1}$ at the indicated date, and subsiding to less that $22 \mathrm{~m} \mathrm{~s}^{-1}$ and veering to southwesterly $12 \mathrm{~h}$ later (according to ECMWF $0.5^{\circ}$ resolution analyses). The storm motion in the direction of wave propagation certainly helped to amplify the local windsea, with a maximum significant wave height $H_{s}$ of $14.1 \mathrm{~m}$ at 0000 UTC on 13 February (according to a numerical wave model configuration that is otherwise verified to produce root mean square errors less than $9 \%$ for $H_{s}>8 \mathrm{~m}$ ).

[43] Using SAR-measured wave periods and directions at different times and locations, we follow great circle trajectories backward at the theoretical group velocity. The location and date of the swell source is defined as the spatial and temporal center of the convergence area and time of the trajectories.

[44] We chose a central peak period, here $15 \mathrm{~s}$, and track the swells forward in space and time, starting from the source center at an angle $\theta_{0}$, following ideal geodesic paths in search of SAR observations. Along each track, SAR data are selected if they are acquired within $3 \mathrm{~h}$ and $100 \mathrm{~km}$ from the theoretical time and position. Great circle tracks are traced from the source in all directions, except for angular sectors with islands.

[45] In order to obtain enough SAR data, we repeat this operation for regularly spaced values of $\theta_{0}$ with a step of $2^{\circ}$. In our case, when varying $\theta_{0}$ from 74 to $90^{\circ}$ (counted clockwise from north), this procedure produced 58 SAR measurements with one swell partition that had a peak 

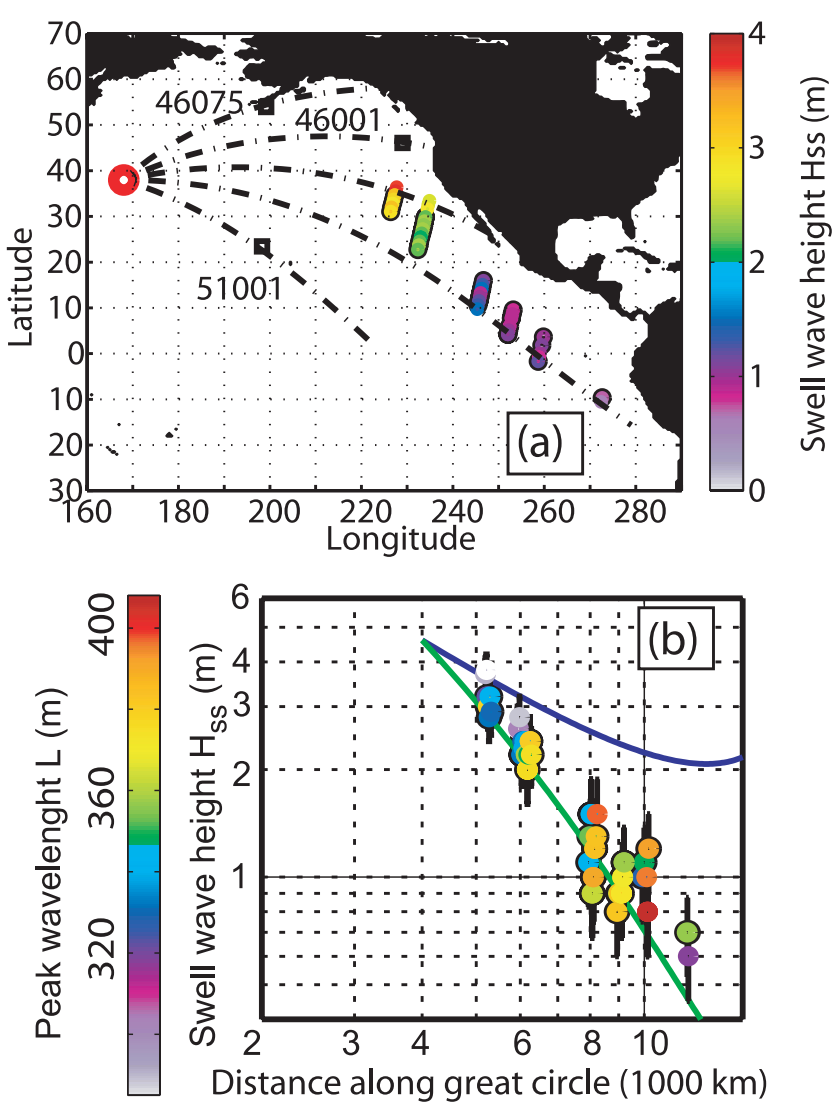

Figure 8. (a) Location of SAR observations with a $15 \mathrm{~s}$ peak period swell system corresponding to the 12 February source, with outgoing directions of 74 to $90^{\circ}$. The same swell was also observed at all buoys from 46075 off western Alaska to 51001 in Hawaii. The dash-dotted line represents great circles leaving the storm source with directions 42, 59, 74, 90 and $106^{\circ}$. (b) Observed swell wave height as a function of distance. The solid lines represent theoretical decays using no dissipation (blue) or the fitted linear dissipation (green) for swells observed in February 2007. Outlined dots are the observations used in the fitting procedure. Error bars show one standard deviation of the expected error on each SAR measurement.

wavelength and direction within $50 \mathrm{~m}$ and $20^{\circ}$ of the expected theoretical value.

[46] If no energy is lost by the wave field, $E_{s}$ decreases asymptotically as $1 /(\alpha \sin (\alpha))$ away from the source. Among the 58 swell observations, we further removed all the data within $4000 \mathrm{~km}$ of the source center, to make sure that the remaining data are in the far field of the storm, and data with a significant swell height $H_{s S}$ less than $0.5 \mathrm{~m}$, after bias correction based on the error model. This makes sure that the signal to noise ratio in the image is large enough so that the wave height estimation is accurate enough.

[47] We then have 35 observations for which we assume that $E_{S}$ is only a function of $\alpha$, and we define the dissipation rate

$$
\mu=-\frac{1}{E_{S} R} \frac{\mathrm{d}\left(E_{S} \alpha \sin \alpha\right)}{\mathrm{d} \alpha} .
$$

Positive values of $\mu$ correspond to losses of wave energy (Figure 8). We then fit an analytical function $H_{s s}(\alpha)$ to the data, defined by a constant $\mu$ and $H_{s s}(\alpha=\pi / 5)$, i.e., the swell height at $4000 \mathrm{~km}$ from the source. Here the couple $H_{s s}(\alpha=\pi / 5)=4.4 \mathrm{~m}$ and $\mu=3.7 \times 10^{-7} \mathrm{~m}^{-1}$ gives the least square difference between the decay with constant $\mu$ and the observed swell decays. Further, the uncertainty of that dissipation rate may be estimated from the known uncertainty of the SAR measurement of $H_{s}$, given by equations (3) and (4). A more simple error model, with larger errors based on the $H_{s 12}$ analysis by Johnsen et al. [2006], does not significantly alter this analysis. Using that error model and neglecting other sources of error in the present analysis, we perturbed the observed swell heights independently to produce a 400 ensemble of synthetic data sets. Taking the $16 \%$ and $84 \%$ levels in the estimation of $\mu$, that would correspond to one standard deviation for a Gaussian distribution, we find that $3.1 \times 10^{-7}<\mu<4.0 \times$ $10^{-7}$ at the $68 \%$ confidence level. This is the first ever estimation of the uncertainty on an observed swell dissipation rate. These values of $\mu$ are more than twice larger than reported by Snodgrass et al. [1966] for smaller amplitude swells.

[48] The formidable height of $4.4 \mathrm{~m}$ at a distance of $4000 \mathrm{~km}$ was observed by the SAR for all outgoing directions from at least 74 to $106^{\circ}$. This same swell was also recorded by buoys in the northeast of Hawaii (NDBC buoy 51001), also with a peak period of $15 \mathrm{~s}$, and a height of $3.4 \mathrm{~m}$ on 16 February 2007 at 0000 UTC. That buoy is located $3300 \mathrm{~km}$ away from the center and in a direction close to $112^{\circ}$. Looking in the northeast quadrant of the storm, one also finds a trace of the swell at buoy 46005, off the Washington coast $\left(4900 \mathrm{~km}\right.$ in direction $\left.59^{\circ}\right)$. There the swell was also observed with a $15 \mathrm{~s}$ period and a maximum height of $3.2 \mathrm{~m}$ on 17 February at $1700 \mathrm{UTC}$, similar to the SAR observations for the same distance. For directions closer to northbound, either the generation was weaker or the Alaskan islands sheltered the coastal buoys. For example, the same swell was also recorded by NDBC buoy number 46075 , off Shumagin Island, Alaska, at a distance of $3000 \mathrm{~km}$ from the source, in the direction $42^{\circ}$. At that buoy, the peak period was $15.0 \mathrm{~s}$ with a maximum swell height of $1.3 \mathrm{~m}$ on 15 February 2008 at 18:30 UTC.

[49] Thus the power radiated by the storm is of the order of $0.5 \mathrm{TW}$ at $4000 \mathrm{~km}$ from the storm center, spread over a $50^{\circ}$ angular sector. This power is about $16 \%$ of the estimated 3.2 TW annual mean flux that reaches the world's coastlines [Rascle et al., 2008]. However, the observed dissipation rate corresponds to an e-folding scale of $3300 \mathrm{~km}$ for the energy. Taking an average propagation distance of $8000 \mathrm{~km}$, only $160 \mathrm{GW}$ would make it to the shore. If the same dissipation rate prevailed closer to the source, then the power radiated at $1000 \mathrm{~km}$ form the storm center was $1.4 \mathrm{TW}$.

[50] We thus expect that the far field dissipation of swells, in spite of the small steepness of these swells, plays a significant role in the air-sea energy balance. This effect probably explains the systematic positive bias for predicted wave heights in wave models that neither account for swell dissipation nor assimilate wave measurements [see, e.g., Rascle et al., 2008].

\section{Conclusions}

[51] Taking advantage of satellite observations of unprecedented coverage and quality, investigations can repeat and 


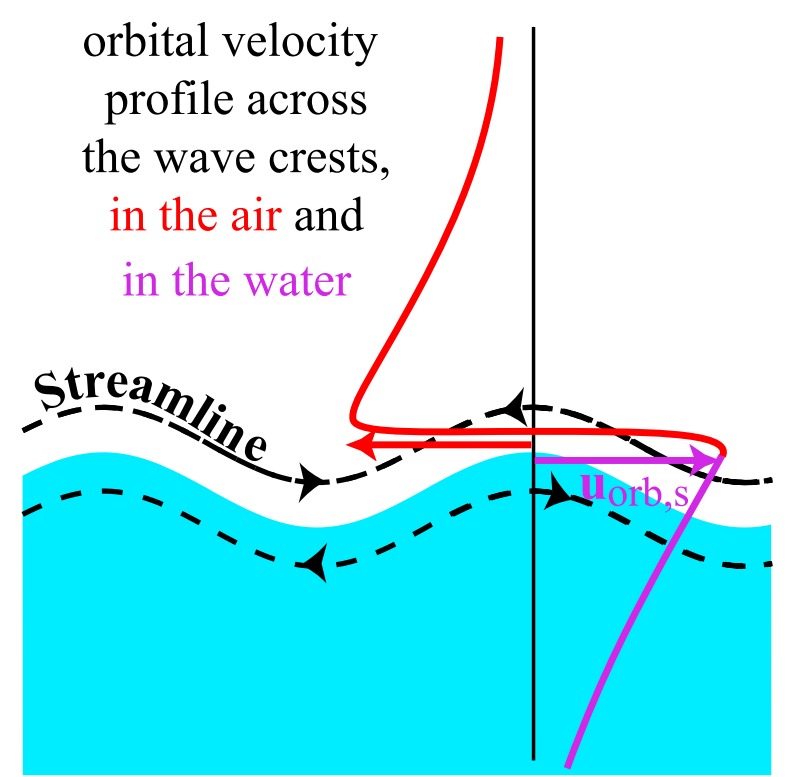

Figure 9. Boundary layer over waves in the absence of wind. Because of the larger inertia of the water compared to the air, most of the adjustment from the subsurface velocity to the free stream velocity in the air occurs on the air side of the surface.

complement the pioneering analysis of swell evolution performed in the 1960s. Severe storms can generate relatively broad spectra of large surface waves. But rapidly, the redistribution of energy, through linear dispersion and nonlinear interaction mechanisms, becomes very effective. The initial wind waves become swells outrunning the wind, leading to the apparition long-crested systems. The propagation properties of these surface gravity waves have been found to closely follow principles of geometrical optics. The consistent patterns of swell fronts dispersing over thousands of kilometers was shown to be useful to provide time series at "virtual wave observing stations," filling gaps in space and time in between the orbit cycles of observation. When compared to buoy measurements, the present results give an explicit dynamical validation of the SAR-derived spectral parameters. As the speed of waves in deep water is proportional to their period or wavelength, information carried by the SAR-derived period and direction distributions, observed at a fairly large distance from the generating area, pertains to the wind conditions existent up to 15 days before.

[52] We also discussed how the swell energy should, in the absence of dissipation, decay in the far field of the storm like $1 /(\alpha \sin \alpha)$ where $\alpha$ is the spherical distance between the storm center and observation point. Exploiting that property allowed us to estimate a dissipation rate $\mu$ of swell energy with unprecedented accuracy, establishing that swell dissipation can be a significant term in the global wave energy budget.

[53] The proposed methodology performed here requires data far enough from the source, typically more than $4000 \mathrm{~km}$, in order to approach this simple asymptotic behavior. At the same time, the swell amplitude should be large enough to be accurately measured by the SAR. Some knowledge of the spectral shape and its spatial distribution inside the storm may be useful to provide better estimates of $\mu$ for lowdissipation cases, or closer to the storm centers. These further analyses will likely benefit from the joint use of data from altimeters, SARs, and other sources of spectral wave information.

[54] A systematic analysis and interpretation of this dissipation is given by Ardhuin et al. [2009a]. These results have also been applied to improve wave forecasting models [Ardhuin et al., 2008, 2009b; Bidlot, 2008]. The parameterization of the dissipation rate could also be used to produce a data-based forecasting system, extending our virtual buoy technique to the estimation of swell heights, with a forward propagation of observations.

[55] Going in the opposite direction, toward the storm source, it is possible that the analysis of swell fields could provide a "new" way of looking into the poorly observed structure of severe storms. Because the usual remote sensing techniques for estimating wind fields either do not work for very high winds or are not well validated [e.g., Quilfen et al., 2006, 2007], the use of far-field swell information may provide an interesting complement to the local wind speeds and wave heights. This inverse problem has already been formulated by Munk et al. [1963] who already proposed an elegant heterodyning technique to push the spatial resolution for the estimation of storm location from swell data, while Heimbach and Hasselmann [2000] have proposed to use wave models to correct wind field errors. The quality of the SAR-derived swell parameters that are coming out of today's Envisat and tomorrow's Sentinel-1, together with a good understanding of the swell energy budget, including its dissipation revealed here, may finally enable this vision.

\section{Appendix A: Viscous Theory for Air-Sea Interaction}

[56] For the sake of simplicity we will consider here the case of monochromatic waves propagating in the $x$ direction only, and we will neglect the curvature of the sea surface. For the small steepness swells considered here that latter approximation is well founded and a more complete analysis is given by Kudryavtsev and Makin [2004]. For deep water waves, the free stream velocity above the waves, just outside of the boundary layer is $u_{+}(x, t)=-\sigma a \cos (k x-\sigma t)$, where $a$ is the swell amplitude and $\sigma=2 \pi / T$ is the radian frequency. The subsurface velocity is $u_{-}(x, t)=\sigma a \cos (k x-\sigma t)$ (Figure 9). Because of the oscillations that propagate at the phase velocity $C$, the horizontal advection of any quantity $X$ by the flow velocity $u$, given by $u \partial X / \partial x$, can be neglected compared to its rate of change in time $\partial X / \partial t$ since the former is a factor $u / C$ smaller than the latter, which is typically less that 0.1 for the swells considered here. Defining $\tilde{u}(x, z, t)=\langle u(x, z, t)\rangle-u-(x, t)$, where the brackets denote an average over flow realizations for a given wave phase. The horizontal momentum equation is thus approximated by,

$$
\frac{\partial \tilde{u}}{\partial t}=-\frac{1}{\rho_{a}} \frac{\partial p}{\partial x}-\frac{\partial u_{-}}{\partial t}+G
$$


where $G$ represents the divergence of the vertical viscous and turbulent fluxes of horizontal momentum,

$$
G=\nu \frac{\partial^{2} \tilde{u}}{\partial z^{2}}+\frac{\partial\left\langle u^{\prime} w^{\prime}\right\rangle}{\partial z}
$$

[57] Because the boundary layer thickness $\delta$ is small compared to the wavelength, the pressure gradient in the boundary layer is given by the pressure gradient above the boundary layer, in balance with the horizontal acceleration. This is another way to write Bernoulli's equation [e.g., Mei, 1989],

$$
-\partial p / \partial x / \rho_{a}=-\sigma^{2} a \sin (k x-\sigma t)=\partial u_{+} / \partial t .
$$

[58] This yields

$$
\frac{\partial \tilde{u}}{\partial t}=2 \frac{\partial u_{+}}{\partial t}+G
$$

with the boundary condition for $z \gg \delta, \tilde{u}$ goes to $2 u_{+}(x, t)$. The equation for the horizontal momentum is thus exactly identical to the one for the oscillatory boundary layer over a fixed bottom with wave of the same period but with an amplitude twice as large. In the viscous case, $G=\nu \partial^{2} \tilde{u} / \partial z^{2}$ and one recovers, after some straightforward algebra, the known viscous result, i.e., for $z>\zeta$,

$$
\begin{aligned}
\tilde{u}(x, z, t)= & 2 \sigma a\left[\mathrm{e}^{-z_{+}} \cos \left(k x-\sigma t-z_{+}\right)-\cos (k x-\sigma t)\right] \\
& +O\left(\rho_{a} / \rho_{w}\right)
\end{aligned}
$$

where $z_{+}=(z-\zeta) / \sqrt{2 \nu / \sigma}$, with the surface elevation $\zeta(x, t)=a \cos (k x-\sigma t)$.

[59] The dissipation rate of energy is given by the mean work of the viscous stresses,

$$
\beta=C_{g} \mu_{v}=\frac{\left\langle\rho_{a} \nu u \partial u / \partial z\right\rangle}{\rho_{w} g a^{2} / 2} .
$$

Equation (A5) gives,

$$
\frac{\partial \tilde{u}}{\partial z}=-2 \sigma a \frac{\mathrm{e}^{-z_{+}}}{\sqrt{2 \nu / \sigma}}\left[\cos \left(k x-\sigma t-z_{+}\right)-\sin \left(k x-\sigma t-z_{+}\right)\right]
$$

This shear provides the low-frequency asymptote to the viscous decay coefficient [Dore, 1978],

$$
\mu_{v}=-2 \frac{\sigma^{2}}{g C_{g}} \frac{\rho_{a}}{\rho_{w}} \sqrt{2 \nu \sigma} \rho_{a} / \rho_{w}
$$

[60] This result was also obtained using a Lagrangian approach without all the above simplifying assumptions [Weber and Forland, 1990, equation (7.1)] in which our $\mu$ correspond to their $2 \alpha$ [Weber and Forland, 1990]. The full viscous result is obtained by also considering the water viscosity $\nu_{w}$, which gives the $O\left(\rho_{a} / \rho_{w}\right)$ correction for the motion in the air, and the classical dissipation term with a decay $\mu_{v w}=-4 k^{2} \nu_{w} / C_{g}$, which dominates for the short gravity waves.

[61] The total dissipation rate is simply $\mu=\mu_{v}+\mu_{v w}$ [Dore, 1978; Weber and Forland, 1990]. For a clean surface with $\nu_{w}=3 \times 10^{-6} \mathrm{~m}^{2} \mathrm{~s}^{-1}, \nu=1.4 \times 10^{-5} \mathrm{~m}^{2} \mathrm{~s}^{-1}$, and $\rho_{a} / \rho_{w}=$ 0.0013 , the two terms are equal for waves with a period $T=$ $1.3 \mathrm{~s}$ and a wavelength of $2.6 \mathrm{~m}$. The air viscosity dominates for all waves longer than this, which is typically the range covered by spectral wave models for sea state forecasting.

[62] For a comparison with fixed bottom boundary layers, the Reynolds number based on the orbital motion should be redefined with a doubled velocity and a doubled displacement, i.e., $\operatorname{Re}=4 u_{\text {orb }} a_{\text {orb }} / \nu$. For monochromatic waves $a_{\text {orb }}=a$ and $u_{\text {orb }}=a \sigma=2 \pi f a / T$. For random waves, investigations of the ocean bottom boundary layer suggest that the boundary layer properties are roughly equivalent to that of a monochromatic boundary layer defined by significant properties [Traykovski et al., 1999].

[63] Although the wind was neglected here, it should influence the shear stresses when its vertical shear is of the order of the wave-induced shear. Taking a boundary layer thickness $\delta$ and wind friction velocity $u_{\star}$, and assuming a logarithmic wind profile, this should occur when $u_{\star} /(\kappa \delta)$ exceeds $2 u_{\text {orb }} / \delta$, where $\kappa$ is von Kármán's constant. This corresponds to, roughly, $u_{\star}>u_{\text {orb }}$. For swells with T $<15 \mathrm{~s}$ and $H_{s s}>2 \mathrm{~m}$ (i.e., $u_{\mathrm{orb}, \mathrm{s}}>0.4 \mathrm{~m} \mathrm{~s}^{-1}$ ), and winds less than $7 \mathrm{~m} \mathrm{~s}^{-1}$ (i.e., $u<0.2 \mathrm{~m} \mathrm{~s}^{-1}$ ), the wind effect on $f_{e}$ may be small and the previous analysis is likely valid. In general, however, the nonlinear interaction of the wave motion and wind should be considered, which requires an extension of existing theories for the distortion of the airflow to finite swell amplitudes.

[64] Finally, the above result for the air viscosity is easily generated to any water depth $D$ by dividing the free stream velocity $u_{+}(x, t)$ by $\tanh (k D)$, so that the dissipation rate is a factor $1 / \tanh ^{2}(k D)$ larger in intermediate water depth.

\section{Appendix B: Quantitative Validation of $\boldsymbol{H}_{s s}$}

[65] A classical analysis of SAR estimation errors is provided by a direct comparison of swell parameters, estimated from level 2 products, with buoy measurements at nearly the same place and time [Holt et al., 1998; Johnsen and Collard, 2004].

[66] Previous validations were presented for the total wave height $H_{s}$ [Collard et al., 2005] or a truncated wave height $H_{s 12}$ defined by chopping the spectrum at a fixed frequency cutoff of $1 / 12 \mathrm{~Hz}$. For that parameter, Johnsen and Collard [2004] found a root mean square (RMS) difference of $0.5 \mathrm{~m}$, when comparing SAR against buoy data, including a bias of $0.2 \mathrm{~m}$. In the present study, we use $H_{s s}$ values obtained from both SAR and buoy spectra.

[67] For each wave spectrum observed in the world ocean, swell partitions are extracted, providing estimations of $H_{s s}, T_{p}$, and $\theta_{p}$. In practice, the L2 spectra are first smoothed over 3 direction bins (i.e., a $30^{\circ}$ sector) and 3 wave number bins, in order to avoid split peaks that would actually correspond to the same swell system. The peaks are then detected and the energy associated to each peak is obtained by the usual inverted water catchment procedure [Gerling, 1992]. The swell peak period is defined as the 


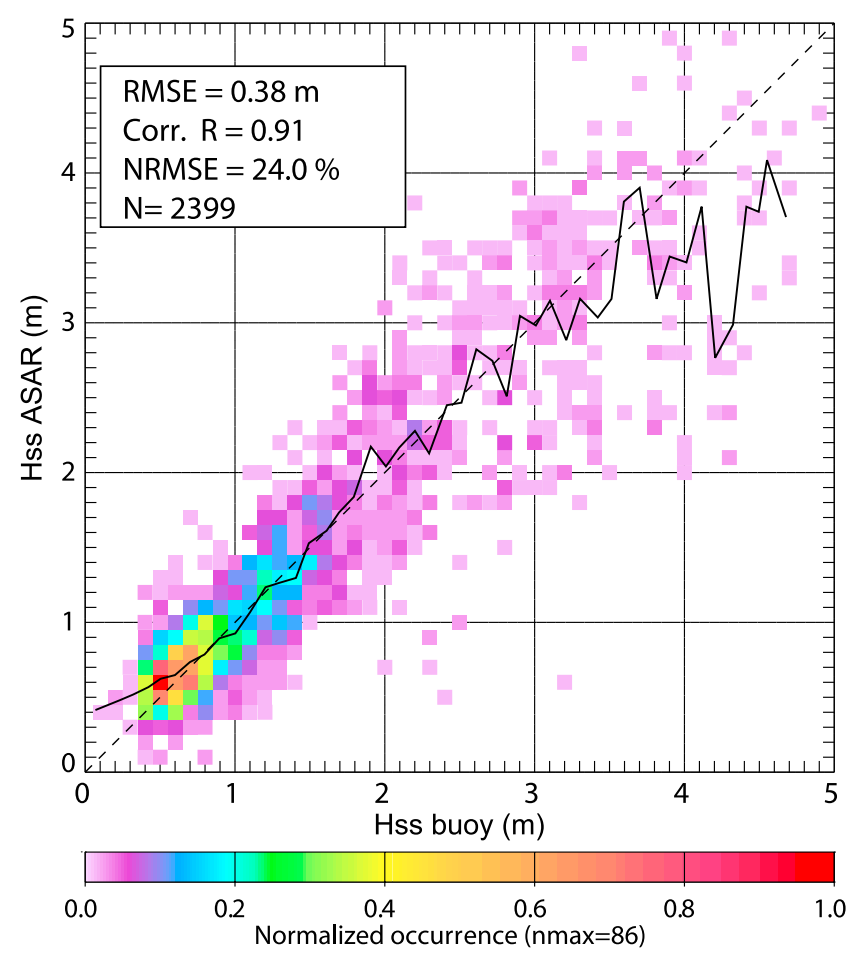

Figure B1. Advanced synthetic aperture radar (ASAR)derived swell partition heights versus buoy swell partition heights after bias correction using equation (3) for subset A. The solid line joins the median values from SAR observations in each $0.1 \mathrm{~m}$ class of buoy-measured height. RMSE, root mean square difference; NRMSE, RMSE normalized by the root mean square observed value.

energy-weighted average around $\pm 22 \%$ of the frequency with the maximum energy. Likewise the peak direction $\theta_{p}$ is defined as the energy-weighted direction within $30^{\circ}$ of the peak direction.

[68] A preliminary validation of $H_{s s}$ was performed by Collard et al. [2006], using L2 processing applied to 4 by $4 \mathrm{~km}$ tiles from narrow swath images exactly located at buoy positions. That study found a $0.37 \mathrm{~m}$ r.m.s. error. This smaller error was obtained in spite of a 4 times smaller image area that should on the contrary produce larger errors because of statistical uncertainties. This suggests that a significant part of the "errors" in SAR validation studies are due to the distance between SAR and buoy observations.

[69] The swell height validation has been repeated here, using all buoy data from 2004 to 2008, located within $200 \mathrm{~km}$ and $1 \mathrm{~h}$ of the SAR observation. These colocated data are made publicly available as part of the XCOL project on the CERSAT ftp server, managed by Ifremer. Because we wished to avoid differences due to coastal sheltering and shallow water effects, we restricted our choice of buoys to distances from the coast and the $100 \mathrm{~m}$ depth contour larger than $100 \mathrm{~km}$. As a result, most selected buoys are not directional, and we use partitions in frequency only. For the present validation, as opposed to sections 2 and 3 of this paper, we thus define a swell partition as the region between two minima of the frequency spectrum. The corresponding energy $E_{s}$ gives the swell height $H_{s s}=4 \sqrt{E_{s}}$. The buoy swell height is then defined from the energy contained within the frequency band of the SAR partition. The peak period is then estimated as the period where the buoy spectrum is maximum. The database includes 15,628 swell partitions observed by the SAR, with matched buoy swell partitions.

[70] Many of these observations correspond to relatively short swells, for which the waves are poorly imaged. We have thus defined a subset of the database by imposing the following conditions. First the image normalized variance, linked to the contrast intensity and homogeneity, should be in the range 1.05 to 1.5 , which limits the data set to 6651 observations. This removes SAR data with nonwave features (slicks, ships..) that would otherwise contaminate the wave spectra. Second, both the SAR and buoy peak periods are restricted to the 12 to $18 \mathrm{~s}$ range, which reduces the data set to 4136 observations, and removes most of the problems related to the azimuthal cutoff. Third and last, the SAR-derived wind speed $U_{10 S A R}$ is limited to range from 3 to $9 \mathrm{~m} \mathrm{~s}^{-1}$ in order to remove low winds with poorly contrasted SAR images and high winds which may still cause some important azimuthal cutoff and contamination of swell spectra by wind sea spectra. This gives subset A, with 2399 observations. The resulting heights are compared to buoy measurements in Figure B1.

[71] When the maximum wind is reduced to $8 \mathrm{~m} \mathrm{~s}^{-1}$, giving subset $\mathrm{B}$, the differences between SAR and buoy data is reduced, with further reductions when the maximum distance between SAR and buoy data is reduced from 200 to $100 \mathrm{~km}$ to give subset C (Table 1).

\section{Appendix C: Derivation and Verification of the Asymptotic Swell Energy Without Dissipation}

\section{C1. Derivation}

[72] The swell energy $E_{s}$ is an integral of the local spectrum $G$ over both frequencies $f$ and arrival directions $\theta$,

$$
E_{s}(\alpha)=\int_{0}^{2 \pi} \int_{0}^{\infty} G\left(t, \phi^{\prime}, \lambda^{\prime}, f, \theta^{\prime}\right) \mathrm{dfd} \theta^{\prime} .
$$

Using equation (1) this local integral, can also be written as an integral over the entire source area $\Omega$. The spherical distance between any point $P(\varphi, \lambda)$ in the source region and the observation point $O(\alpha, 0)$ is $\alpha^{\prime}$. The observed frequency that is due to this source point is

$$
f=g t /\left(4 \pi R \alpha^{\prime}\right)=f_{0} \frac{\alpha}{\alpha^{\prime}} .
$$

We may replace $f$ by $\alpha^{\prime}$ in equation (C1),

$$
E_{S}(\alpha)=\frac{f_{0}}{\alpha} \int_{0}^{2 \pi} \int \frac{\alpha^{2} G\left(t_{0}, \phi, \lambda, f, \theta\right)}{\alpha^{\prime 2}} \mathrm{~d} \theta^{\prime} \mathrm{d} \alpha^{\prime} .
$$

For a circular uniform storm of radius $r$ with isotropic spectra, as used in Figure $\mathrm{C} 1, E_{s}(\alpha)$ is given by the integral 
Table 1. Error Statistics for Swell Partitions' Heights and Peak Periods Derived From SAR Wave Mode Data (After Bias Correction) Against Buoy-Derived Data for Subsets A, B, and C of the Colocated Database ${ }^{\mathrm{a}}$

\begin{tabular}{lcc}
\hline & $H_{s S}$ & $T_{p}$ \\
\hline & Subset $A$ & \\
Bias & $0.00 \mathrm{~m}$ & $0.27 \mathrm{~s}$ \\
RMSE & $0.38 \mathrm{~m}$ & 1.14 \\
SI & $24.0 \%$ & $7.9 \%$ \\
NRMSE & $24.0 \%$ & $8.2 \%$ \\
$r$ & 0.91 & 0.61 \\
& Subset B & \\
Bias & $0.00 \mathrm{~m}$ & $0.24 \mathrm{~s}$ \\
RMSE & $0.35 \mathrm{~m}$ & $1.11 \mathrm{~s}$ \\
SI & $23.5 \%$ & $7.8 \%$ \\
NRMSE & $23.5 \%$ & $8.0 \%$ \\
$r$ & 0.92 & 0.62 \\
& Subset C & $0.32 \mathrm{~s}$ \\
Bias & $0.02 \mathrm{~m}$ & $1.07 \mathrm{~s}$ \\
RMSE & $0.29 \mathrm{~m}$ & $7.3 \%$ \\
SI & $22.4 \%$ & $7.7 \%$ \\
NRMSE & $22.5 \%$ & 0.64 \\
$r$ & 0.92 & \\
\hline
\end{tabular}

${ }^{a}$ Subset A contains 2399 observations. Subset B is restricted to $U_{10 S A R} \leq$ $8 \mathrm{~m} \mathrm{~s}^{-1}$ and contains 1936 observations. Subset $\mathrm{C}$ is further restricted to SAR-buoy distances less than $100 \mathrm{~km}$ and contains only 460 observations. RMSE, root mean square difference; NRMSE, RMSE normalized by the root mean square observed value; SI, scatter index. Scatter index is equivalent to the NRMSE computed after bias removal. Finally, $r$ is Pearson's linear correlation coefficient.

over $\alpha^{\prime}$ weighted by the directional width of the spectrum $\Delta \theta^{\prime}$. That width is given by the spherical trigonometry relationship

$$
\Delta \theta^{\prime}=2 \theta_{\max }^{\prime}=2 \arccos \left[\frac{\cos \varphi_{\max }-\cos \alpha \cos \alpha^{\prime}}{\sin \alpha \sin \alpha^{\prime}}\right]
$$

with $\varphi_{\max }=r / R$.

[73] For general spectral distribution, we may transform the integration variables $\left(\alpha^{\prime}, \theta^{\prime}\right)$ which are the colatitude and longitude coordinates on the sphere with a pole at the observation point, to coordinates $(\varphi, \lambda)$ with a pole in the center of the swell field at $t_{0}$. The transformation Jacobian is simply given by the equality of the elementary area on the unit radius sphere $d A=\sin \varphi \mathrm{d} \phi \mathrm{d} \lambda=|\cos \phi| \mathrm{d} \phi \mathrm{d} \lambda=$ $\sin \alpha^{\prime} \mathrm{d} \alpha^{\prime} \mathrm{d} \theta^{\prime}$. We thus have

$$
\begin{aligned}
E_{S}(\alpha)= & \frac{f_{0}}{\alpha \sin \alpha} \int_{\Omega} \frac{\alpha^{2} \sin \alpha}{\alpha^{\prime 2} \sin \alpha^{\prime}} G\left(t_{0}, \phi, \lambda, f, \theta\right)|\cos \phi| \mathrm{d} \phi \mathrm{d} \lambda \\
= & \frac{f_{0}}{\alpha \sin \alpha}\left\{\int_{\Omega} G\left(t_{0}, \phi, \lambda, f_{0}, \theta_{0}\right) d A\right. \\
& \times\left[1+O\left(\frac{\Delta_{\alpha}}{\alpha}\right)\right] \\
& +\int_{\Omega}\left[G\left(t_{0}, \phi, \lambda, f, \theta\right)-G\left(t_{0}, \phi, \lambda, f_{0}, \theta\right)\right] \mathrm{d} A \\
& \left.+\int_{\Omega}\left[G\left(t_{0}, \phi, \lambda, f_{0}, \theta\right)-G\left(t_{0}, \phi, \lambda, f_{0}, \theta_{0}\right)\right] \mathrm{d} A\right\}
\end{aligned}
$$

where $\theta$ is the direction of the great circle at the generation point that goes through that point and the observation point. $\theta$ is thus a function of $\phi, \lambda, \alpha$ and $\theta_{0} . \Delta_{\alpha}$ is maximum value of $\left|\alpha^{\prime}-\alpha\right|$, i.e., the radius of the source region divided by the Earth radius.

[74] For continuous spectra, the second integral on the right hand side of equation $(\mathrm{C} 1)$ goes to zero as $\alpha$ goes to infinity (on the sphere, however, $\alpha$ is always less than $\pi$, but we shall see that $\pi$ is large enough) since the part of the source spectra that contribute to the observations shrink to a smaller and smaller neighborhood around $f_{0}$ and $\theta_{0}$. The observed frequencies $f$ are limited by

$$
\frac{\left|f-f_{0}\right|}{f_{0}} \leq \frac{\Delta_{\alpha}}{\alpha}
$$

This is enough to guarantee that this second integral also contributes a deviation $\varepsilon_{2}$ from the asymptote, limited by

$$
\varepsilon_{2} \leq A \frac{\Delta_{\alpha}}{\alpha} f_{0} \max \left\{\frac{\partial G}{\partial f}\right\}
$$

where the maximum is taken over all the contributing components.

[75] Similarly, the outgoing directions $\theta$ received at the observation point are also limited to a narrow window as $\alpha$ increases, giving another deviation term $\varepsilon_{3}$. Using the sine formula in the triangle $O P N, \sin \theta / \sin (\pi-\alpha)=\sin \lambda / \sin \alpha^{\prime}$. Thus, in the far field of the storm and its antipode, $\theta$ is close to $\theta_{0}=\lambda$. Thus $\sin \theta-\sin \theta_{0}=\sin \lambda\left(\sin \alpha-\sin \alpha^{\prime}\right) / \sin \alpha^{\prime}$, which is less than $\Delta_{\alpha} / \sin \alpha^{\prime}$, and therefore $\left|\theta-\theta_{0}\right|$ is less than $\arcsin \left(\Delta_{\alpha} / \sin \alpha^{\prime}\right)$. If one does not get too close to the

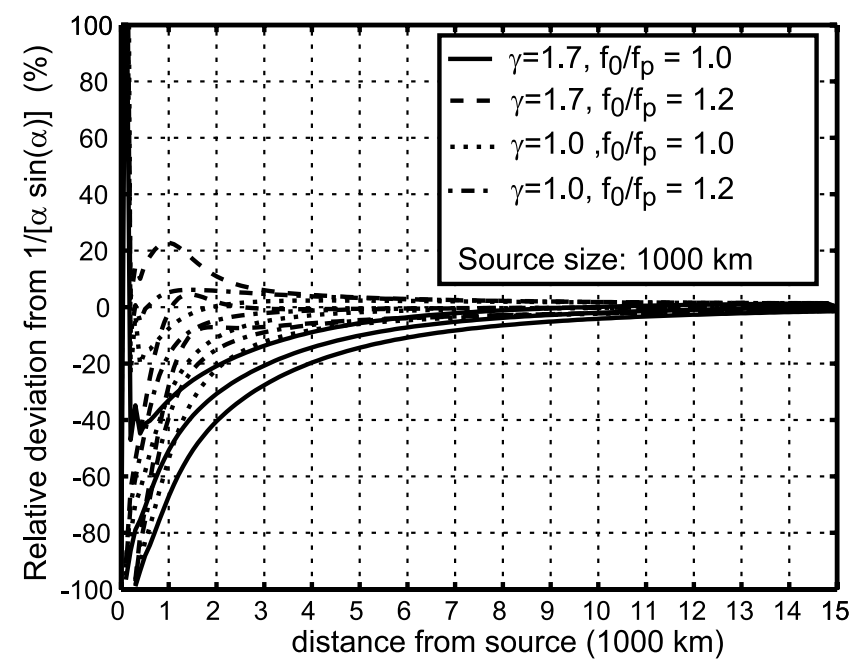

Figure C1. Convergence of the swell energy $E_{s}$ integral (C1) toward the asymptote $1 /(\alpha \sin \alpha)$, as a function of spectrum width for a storm diameter of $1000 \mathrm{~km}$. The result is independent of the choice of $f_{p}$. In practice the calculations were made for $f_{p}=0.07 \mathrm{~Hz}\left(T_{p}=13 \mathrm{~s}\right)$. The three lines for each case correspond to position errors $\Delta_{x}$ of $-200,0$, and $200 \mathrm{~km}$ relative to the great circle trajectory. For all cases considered here, the deviation is less than $20 \%$ beyond $4000 \mathrm{~km}$ from the storm center. 
storm or its antipode (say, $\Delta_{\alpha}<\alpha<\pi-2 \Delta_{\alpha}$ ) then we can give an upper bound of $1 / \sin \alpha^{\prime}$ as a function of $\alpha$ and obtain

$$
\varepsilon_{3} \leq A \arcsin \left(2 \frac{\Delta_{\alpha}}{\sin \alpha}\right) \max \left\{\frac{\partial G}{\partial \theta}\right\}
$$

The deviation from the asymptote due to $\varepsilon_{3}$ is thus of the order of $\Delta_{\alpha} / \sin \alpha$ and may increase close to the antipode, if waves from a wide range of directions can reach that point. In practice this does not happen since continents and island chains block most of the arrival directions at the antipode, leaving only a small window of possible arrival directions [e.g., Munk et al., 1963]. Directional wave spectra in active generation areas are generally relatively broad with $\partial G / \partial \theta /$ $G$ typically less than 2 for directions within $30^{\circ}$ of the main wave direction. On the contrary, typical storm spectra can give $f \partial G / \partial f / G$ as large as 10 for frequencies within $30 \%$ of the peak frequency. We thus expect the deviation $\varepsilon_{2}$ to dominate over $\varepsilon_{3}$.

\section{C2. Verification}

[76] For a useful comparison with observations, the asymptotic swell height evolution should be approached on a scale smaller than the ocean basin scale. This is easily tested for storms with spatially uniform spectra over a radius $r$, by evaluating the integral in equation (C3). We chose a center frequency $f_{0}$ and consider the swell energy at a distance $R \alpha+\Delta_{x}$ and a time $t(\alpha)$ such that $R \alpha=g t /\left(4 \pi f_{0}\right)$. $\Delta_{x}$ is thus an error relative to the theoretical position of the wave group of frequency $f_{0}$. The relative difference of $E_{s}(\alpha)$ and its asymptotic evolution $1 /(\alpha \sin \alpha)$ depends only on the spectral shape in the storm, the relative frequency $f_{0} / f_{p}$ where $f_{p}$ is the peak frequency, the distance $\alpha$, the storm size $\alpha /(r / R)$, and the position error $\Delta_{x}$. Results are shown in Figure $\mathrm{C} 1$ for isotropic directional spectra, in which case $\varepsilon_{3}=0$. Although an isotropic spectrum is not realistic at all, it allows for simple calculations. As discussed below, the effect of directional spreading is expected to be less important than the shape of the frequency spectrum.

[77] As indicated by equation (C8), the contribution of both the spectral shape and $f_{0} / f_{p}$ comes through the maximum relative variation of $F$ in the frequency interval that contribute to $E_{s}$. Here we take the spectra in the storm to have a JONSWAP shape [Hasselmann et al., 1973], that we adjust by varying the peak enhancement factor $\gamma$. A relatively broad [Pierson and Moskowitz, 1964] spectrum is obtained with $\gamma=1$. If we chose $f_{0}=f_{p}$, this spectrum will give smaller deviations of $E_{s}$ from the asymptote (solid lines in Figure C1), than narrower spectra with larger values of $\gamma$. Young [2005] showed that wave spectra in Hurricanes generally fall in between these two categories, with a typical value $\gamma_{J}=1.7$. Larger deviations from the asymptote are obtained for $f_{0}<f_{p}$, since the forward face of the spectrum is very steep, while smaller errors are obtained for $f_{0}>f_{p}$ because of the more gentle decrease of $F$ toward the high frequencies.

[78] Similarly, large deviations are produced if the observations are made in a direction far from the peak generation direction in cases when the directional spectrum is narrow. For observation directions $30^{\circ}$ from the peak direction, and spectra with $\cos ^{4}(\theta)$ directional distribution, the deviations are still dominated by the dispersive term $\varepsilon_{2}$, as expected.

[79] The other important factor is the distance $\alpha$ relative to the storm size $\varphi_{\max }=r / R$. A faster convergence is obtained for smaller storms. If observations do not correspond exactly to a theoretical propagation at a group speed $C_{g}$ but are within a distance $\Delta_{x}$ of the theoretical position, the values of $E_{s}$ will also be affected in a way that depends on the spectral shape. In the absence of energy gains or losses, and for realistic storm sizes and spectral shapes, the deviation of observations from the asymptote should be less than $20 \%$ for $x>4000 \mathrm{~km}$ when $\Delta_{x}<200 \mathrm{~km}$ is enforced.

[80] Acknowledgments. SAR data were provided by the European Space Agency (ESA), and buoy data were kindly provided by the U.S. National Data Buoy Center and Marine Environmental Data Service of Canada. ESA funded the XCOL colocated database and the initial work on the virtual buoy concept. The swell decay analysis was funded by the French Navy as part of the EPEL program. This work is a contribution to the ANR-funded project HEXECO and DGA-funded project ECORS.

\section{References}

Aouf, L., J.-M. Lefèvre, D. Hauser, and B. Chapron (2006), On the combined assimilation of RA-2 altimeter and ASAR wave data for the improvement of wave forecasting, paper presented at 15 Years of Radar Altimetry Symposium, Eur. Space Agency, Venice, Italy.

Ardhuin, F., and A. D. Jenkins (2006), On the interaction of surface waves and upper ocean turbulence, J. Phys. Oceanogr., 36, 551-557.

Ardhuin, F., F. Collard, B. Chapron, P. Queffeulou, J.-F. Filipot, and M. Hamon (2008), Spectral wave dissipation based on observations: A global validation, paper presented at Chinese-German Joint Symposium on Hydraulics and Ocean Engineering, Tech. Univ. Darmstadt, Darmstadt, Germany.

Ardhuin, F., B. Chapron, and F. Collard (2009a), Observation of swell dissipation across oceans, Geophys. Res. Lett., 36, L06607, 10.1029/ 2008GL037030.

Ardhuin, F., L. Marié, N. Rascle, P. Forget, and A. Roland (2009b), Observation and estimation of Lagrangian, Stokes and Eulerian currents induced by wind and waves at the sea surface, J. Phys. Oceanogr, doi:10.1175/2009JPO4169.1, in press.

Bidlot, J.-R. (2008), Intercomparison of operational wave forecasting systems against buoys: Data from ECMWF, MetOffice, FNMOC, NCEP, DWD, BoM, SHOM and JMA, September 2008 to November 2008, technical report, Joint WMO-IOC Tech. Comm. for Oceanogr. and Mar. Meteorol., Paris. (Available at http://www.jcomm-services.org/modules/ documents/documents/modelcomparisonsecondlist200809200811.pdf)

Chapron, B., H. Johnsen, and R. Garello (2001), Wave and wind retrieval from SAR images of the ocean, Ann. Telecommun., 56, 682-699.

Collard, F., F. Ardhuin, and B. Chapron (2005), Extraction of coastal ocean wave fields from SAR images, IEEE J. Oceanic Eng., 30(3), 526-533.

Collard, F., B. Chapron, F. Ardhuin, H. Johnsen, and G. Engen (2006), Coastal ocean wave retrieval from ASAR complex images, paper presented at OceanSAR, Earth Obs. Mar. Surveillance Coord. Comm., Saint John's, Nfld. Lab., Canada.

Darbyshire, J. (1958), The generation of waves by wind, Philos. Trans. $R$ Soc. London, Ser. A, 215, 299-328.

Dore, B. D. (1978), Some effects of the air-water interface on gravity waves, Geophys. Astrophys. Fluid Dyn., 10, 215-230.

Engen, G., and H. Johnsen (1995), SAR-ocean wave inversion using image cross spectra, IEEE Trans. Geosci. Remote Sens., 33, 1047-1056.

Fabrikant, A. L., and M. A. Raevsky (1994), The influence of drift flow turbulence on surface gravity wave propagation, J. Fluid Mech., 262, $141-156$.

Gerling, T. W. (1992), Partitioning sequences and arrays of directional ocean wave spectra into component wave systems, J. Atmos. Oceanic Technol., 9, 444-458.

Gjevik, B., H. E. Korgstad, A. Lygre, and O. Rygg (1988), Long period swell wave events on the Norwegian shelf, J. Phys. Oceanogr., 18, 724-737.

Grachev, A. A., and C. W. Fairall (2001), Upward momentum transfer in the marine boundary layer, J. Phys. Oceanogr., 31, 1698-1711.

Hanson, J. L., and O. M. Phillips (2001), Automated analysis of ocean surface directional wave spectra, J. Atmos. Oceanic Technol., 18, 277-293.

Hasselmann, K. (1963), On the non-linear energy transfer in a gravity-wave spectrum. Part 3. Evaluation of the energy flux and swell-sea interaction for a Neuman spectrum, J. Fluid Mech., 15, 385-398. 
Hasselmann, K., et al. (1973), Measurements of wind-wave growth and swell decay during the Joint North Sea Wave Project, Dtsch. Hydrogr. Z., 8(12), suppl. A, 1-95.

Hasselmann, S., C. Brüning, and K. Hasselmann (1996), An improved algorithm for the retrieval of ocean wave spectra from synthetic aperture radar image spectra, J. Geophys. Res., 101, 16,615-16,629.

Hasselmann, S., P. Lionello, and K. Hasselmann (1997), An optimal interpolation scheme for the assimilation of spectral wave data, J. Geophys. Res., 102, 15,823-15,836.

Heimbach, P., and K. Hasselmann (2000), Development and application of satellite retrievals of ocean wave spectra, in Satellites, Oceanography and Society, edited by D. Halpern, pp. 5-33, Elsevier, Amsterdam.

Holt, B., A. K. Liu, D. W. Wang, A. Gnanadesikan, and H. S. Chen (1998), Tracking storm-generated waves in the northeast Pacific Ocean with ERS-1 synthetic aperture radar imagery and buoys, J. Geophys. Res., 103, 7917-7929.

Johnsen, H., and F. Collard (2004), ASAR wave mode processing Validation of reprocessing upgrade, Tech. Rep. 168, North. Res. Inst., Troms, Norway.

Johnsen, H., G. Engen, F. Collard, V. Kerbaol, and B. Chapron (2006), Envisat ASAR wave mode products - Quality assessment and algorithm upgrade, in Proceedings of SEASAR 2006, Eur. Space Agency Spec. Publ., ESA SP-613.

Kedar, S., M. Longuet-Higgins, F. W. N. Graham, R. Clayton, and C. Jones (2008), The origin of deep ocean microseisms in the North Atlantic Ocean, Proc. R. Soc., Ser. A, 1-35, doi:10.1098/rspa.2007.0277.

Kerbaol, V., B. Chapron, and P. Vachon (1998), Analysis of ERS-1/2 synthetic aperture radar wave mode imagettes, J. Geophys. Res., 103, 7833-7846.

Krogstad, H. E. (1992), A simple derivation of Hasselmann's nonlinear ocean-synthetic aperture radar transform, J. Geophys. Res., 97, 24212425 .

Kudryavtsev, V. N. (1994), The coupling of wind and internal waves, J. Fluid Mech., 278, 33-62.

Kudryavtsev, V. N., and V. K. Makin (2004), Impact of swell on the marine atmospheric boundary layer, J. Phys. Oceanogr., 34, 934-949.

Mei, C. C. (1989), Applied Dynamics of Ocean Surface Waves, 2nd ed., 740 pp., World Sci., Singapore.

Munk, W. H., and M. A. Traylor (1947), Refraction of ocean waves: A process linking underwater topography to beach erosion, J. Geol., $L V(1)$, $1-26$.

Munk, W. H., G. R. Miller, F. E. Snodgrass, and N. F. Barber (1963), Directional recording of swell from distant storms, Philos. Trans. $R$. Soc. London, Ser. A, 255, 505-584.

Pierson, W. J., Jr., and L. Moskowitz (1964), A proposed spectral form for fully developed wind seas based on the similarity theory of S. A. Kitaigorodskii, J. Geophys. Res., 69, 5181-5190.
Quilfen, Y., J. Tournadre, and B. Chapron (2006), Altimeter dual-frequency observations of surface winds, waves, and rain rate in tropical cyclone Isabel, J. Geophys. Res., 87, C01004, 10.1029/2005JC003068.

Quilfen, Y., C. Prigent, B. Chapron, A. A. Mouche, and N. Houti (2007) The potential of QuikSCAT and WindSat observations for the estimation of sea surface wind vector under severe weather conditions, J. Geophys. Res., 112, C09023, 10.1029/2007JC004163.

Rascle, N., F. Ardhuin, P. Queffeulou, and D. Croizé-Fillon (2008), A global wave parameter database for geophysical applications. Part 1: Wave-current-turbulence interaction parameters for the open ocean based on traditional parameterizations, Ocean Modell., 25, 154-171, doi:10.1016/j.ocemod.2008.07.006.

Schulz-Stellenfleth, J., S. Lehner, and D. Hoja (2005), A parametric scheme for the retrieval of two-dimensional ocean wave spectra from synthetic aperture radar look cross spectra, J. Geophys. Res., 110, C05004, 10.1029/2004JC002822.

Schulz-Stellenfleth, J., T. König, and S. Lehner (2007), An empirical approach for the retrieval of integral ocean wave parameters from synthetic aperture radar data, J. Geophys. Res., 21, C03019, 10.1029/ 2006JC003970.

Snodgrass, F. E., G. W. Groves, K. Hasselmann, G. R. Miller, W. H. Munk, and W. H. Powers (1966), Propagation of ocean swell across the Pacific, Philos. Trans. R. Soc. London, Ser. A, 249, 431-497.

Tolman, H. L. (2002), Alleviating the garden sprinkler effect in wind wave models, Ocean Modell., 4, 269-289.

Traykovski, P., A. E. Hay, J. D. Irish, and J. F. Lynch (1999), Geometry, migration, and evolution of wave orbital ripples at LEO-15, J. Geophys. Res., 104, 1505-1524.

Waves in Shallow Water Environment (WISE) Group (2007), Wave modelling — The state of the art, Prog. Oceanogr., 75, 603-674, doi:10.1016/j.pocean.2007.05.005.

Weber, J. E., and E. Førland (1990), Effect of the air on the drift velocity of water waves, J. Fluid Mech., 218, 619-640.

Young, I. R. (2005), Directional spectra of hurricane wind waves, J. Geophys. Res., 111, C08020, 10.1029/2006JC003540.

F. Ardhuin, Service Hydrographique et Océanographique de la Marine, Technopole Brest Iroise, F-29609 Brest, France. (ardhuin@shom.fr)

B. Chapron, Laboratoire d'Océanographie Spatiale, Centre de Brest, Ifremer, 13 Rue du Chatellier, F-29280 Plouzané, France. (bertrand.chapron@ ifremer.fr)

F. Collard, Division Radar, Collecte Localisation Satellites, Batiment Ponant, Avenue la Pèrouse, Technopole Brest Iroise, F-29280 Plouzané, France. (dr.fab@cls.fr) 